\title{
Hiperciclicidade em espaços de funções inteiras
}

\author{
André Arbex Hallack
}

TESE APRESENTADA AO

INSTITUTO DE MATEMÁTICA E ESTATÍSTICA DA

UNIVERSIDADE DE SÃO PAULO PARA

OBTENÇÃO DO GRAU DE

DOUTOR EM CIÊNCIAS (MATEMÁTICA)

\author{
Área de Concentração: Análise \\ Orientadora: Prof. ${ }^{a}$ Mary Lilian Lourenço \\ Co-orientador: Prof. Richard M. Aron
}

Durante a elaboração deste trabalho o autor recebeu apoio financeiro da CAPES

- São Paulo, novembro de 2005 - 


\section{Hiperciclicidade em espaços de funções inteiras}

Este exemplar corresponde à redação final

(corrigida) da tese de doutoramento

defendida por André Arbex Hallack

e aprovada pela comissão julgadora.

São Paulo, 11 de novembro de 2005.

Banca examinadora:

- Profa. Dra. Mary Lilian Lourenço (Orientadora) - IME - USP

- Prof. Dr. Richard Martin Aron (Co-orientador) - KENT STATE UNIVERSITY

- Prof. Dr. Raymundo Luiz Alencar - CTA - ITA

- Prof. Dr. Geraldo Márcio de Azevedo Botelho - UFU

- Prof. Dr. Nilson da Costa Bernardes Junior - UFF 
À minha familia. 


\section{Agradecimentos}

À professora Mary Lilian Lourenço, pela dedicação e paciência com que me orientou ao longo do doutorado.

Ao professor Richard M. Aron, pela orientação e pela forma acolhedora com que, juntamente com sua esposa, me recebeu em Kent.

Aos colegas do Departamento de Matemática da Universidade Federal de Juiz de Fora, pelo afastamento concedido.

Aos colegas do IME-USP e da Kent State University, pelo convívio amigável e que tanto me ajudou no cumprimento de mais esta etapa.

À minha tia Lucy, pelo apoio e carinho com que me recebeu desde o início deste doutoramento em São Paulo.

À minha família, pelo apoio em todos os momentos. 


\section{Resumo}

A proposta deste trabalho é estudar, sob vários aspectos, o fenômeno da Hiperciclicidade para operadores em espaços de funções inteiras. Iniciamos obtendo uma prova simples de que o conjunto das funções hipercíclicas comuns a todas as translações por um complexo não-nulo em $H(\mathbb{C})$ é "lineável". Fornecemos também uma prova completa da hiperciclicidade de uma classe de exemplos de operadores que não são de convolução. Investigamos o tamanho do conjunto de vetores hipercíclicos, num sentido topológico, para certos operadores, através da introdução de um conceito de certa forma similar ao conceito de porosidade para espaços métricos. Finalmente, adaptando o Teorema de Runge para que tenhamos um resultado que funcione sobre certos domínios em qualquer espaço de Banach, estendemos resultados relativos à hiperciclicidade de translações em $H(\mathbb{C})$ para subespaços de $H_{b}(E)$ (em certos casos todo o $H_{b}(E)$ ), podendo $E$ pertencer a uma vasta classe de espaços de Banach.

\section{Abstract}

The purpose of this work is to study some aspects of Hypercyclicity related to operators on spaces of entire functions. We begin with a simple proof, showing that the set of common hypercyclic functions for the translations on $H(\mathbb{C})$ is "lineable". We also give a complete proof for the hypercyclicity of a class of non-convolution operators. Introducing a concept somehow similar to the notion of porosity in metric spaces, we then investigate the size (in a topological sense) of the set of hypercyclic vectors for some operators. Finally, we adapt Runge's Theorem to work on certain domains in any Banach space and extend some results on the hypercyclicity of translations on $H(\mathbb{C})$ to subspaces of $H_{b}(E)$ (in some cases all of $H_{b}(E)$ ), $E$ being in a large class of Banach spaces. 


\section{Índice}

1 Preliminares 1

2 Uma observação sobre "lineabilidade" $\quad 19$

3 Operadores hipercíclicos que não são de convolução 25

4 Sobre porosidade e hiperciclicidade $\quad 31$

5 Hiperciclicidade para translações através do Teorema de Runge 41

$\begin{array}{ll}\text { Algumas notações e definições } & 61\end{array}$

Referências $\quad 65$ 


\section{Capítulo 1}

\section{Preliminares}

\section{Famílias universais: definições e resultados básicos}

Ao longo dos tempos têm sido obtidos diversos exemplos de processos limites em Análise que divergem de maneira maximal. Esta divergência maximal está frequentemente associada ao fenômeno da UNIVERSALIDADE (existência de um único objeto, o qual nos permite aproximar toda uma classe de objetos). Vamos destacar alguns exemplos históricos para ilustrar esta afirmação:

- 1914 - Fekete [33]: existe uma série formal real de potências $\sum_{n=1}^{\infty} a_{n} x^{n}$ em $[-1,1]$ que não apenas diverge em todo ponto $x \neq 0$, mas também o faz de modo que, para cada função contínua $g$ em $[-1,1]$ com $g(0)=0$, é possível obter uma sequência crescente $\left(n_{k}\right)$ de naturais tal que $\sum_{n=1}^{n_{k}} a_{n} x^{n} \longrightarrow g(x)$ uniformemente!

- 1929 - Birkhoff [13]: no espaço $H(\mathbb{C})$ de funções inteiras, existe uma função inteira $f$ tal que, para cada função inteira $g$ é possível obter uma sequência $\left(a_{n}\right)$ de complexos com $f\left(z+a_{n}\right) \longrightarrow g(z)$ uniformemente em cada compacto de $\mathbb{C}$ ! 
- 1952 - MacLane [28]: no espaço $H(\mathbb{C})$ de funções inteiras, existe uma função inteira $f$ tal que, para cada função inteira $g$ é possível obter uma sequência crescente $\left(n_{k}\right)$ de naturais com $f^{\left(n_{k}\right)}(z) \longrightarrow g(z)$ uniformemente em cada compacto de $\mathbb{C}$ !

- 1969 - Rolewicz [34]: no espaço de Banach $E=\ell_{p}(1 \leq p<\infty)$ ou $E=c_{0}$, consideremos o operador $T: E \rightarrow E$ dado por $T\left(x_{1}, x_{2}, x_{3}, \ldots\right)=\left(a x_{2}, a x_{3}, a x_{4}, \ldots\right)$ (weighted backward shift), com $|a|>1$. Existe um vetor $x \in E$ tal que sua "órbita" sob $T$, dada por $\mathcal{O}(x ; T)=\left\{x, T x, T^{2} x, \ldots\right\}$, é densa em $E$ !

Definimos o fenômeno da universalidade, de uma forma bem geral, do seguinte modo:

Definição 1.1. Sejam $X$ e $Y$ espaços topológicos e $T_{i}: X \rightarrow Y(i \in I)$ uma coleção (geralmente enumerável) de aplicações contínuas. Um elemento $x \in X$ é dito UNIVER$S A L$ (para a familia $\left(T_{i}\right)_{i \in I}$ ) quando o conjunto $\left\{T_{i} x ; i \in I\right\}$ é denso em $Y$.

Devido ao caráter muito geral da definição acima, resultados totalmente gerais sobre universalidade são raros. Desta forma, o usual é o estudo do fenômeno em diversos "ambientes", dando origem a vários "tipos de universalidade": operadores hipercíclicos (um caso especial no "ambiente linear"), séries de potências e séries de Taylor universais (no contexto tanto da Análise Real quanto da Análise Complexa), primitivas universais, etc.

Também no nível dos objetos a universalidade é abundante, se existente: em muitos casos quase todo elemento é universal (no sentido de categorias de Baire).

O primeiro resultado que vamos destacar, devido a Grosse-Erdmann, é o ...

Teorema 1.2. (Critério de Universalidade [25]) Sejam $X$ um espaço de Baire, $Y$ um espaço "segundo-contável" (ou seja, cuja topologia admite base enumerável de abertos) $e$ $T_{i}: X \rightarrow Y(i \in I)$ uma coleção de aplicações contínuas. Se denotarmos por $\mathcal{U} \subset X \quad$ o conjunto dos elementos universais relativos à familia $\left(T_{i}\right)$, são equivalentes:

(a) $O$ conjunto $\mathcal{U}$ é residual em $X\left(\mathcal{U}\right.$ contém um conjunto $G_{\delta}$ denso em $\left.X\right)$;

(b) $O$ conjunto $\mathcal{U}$ é denso em $X$;

(c) Para cada par de abertos não-vazios $U \subset X$ e $V \subset Y$, é possivel obter algum índice 
$i \in I$ tal que

$$
T_{i}(U) \cap V \neq \phi
$$

Se estas condições são verdadeiras, então $\mathcal{U}$ é um conjunto $G_{\delta}$ denso em $X$.

Demonstração:

Seja $\mathcal{B}=\left\{V_{n} ; n \in \mathbb{N}\right\}$ uma base de abertos para a topologia de $Y$.

Então:

$$
\mathcal{U}=\bigcap_{n \in \mathbb{N}} A_{n} \text {, sendo } A_{n}=\bigcup_{i \in I} T_{i}^{-1}\left(V_{n}\right) \text { (aberto) para todo } n \in \mathbb{N} \text {. }
$$

De fato: Seja $x \in \mathcal{U}$. Dado $n \in \mathbb{N}$, existe $k \in I$ tal que $T_{k}(x) \in V_{n}$. Logo $x \in T_{k}^{-1}\left(V_{n}\right) \subset A_{n}$ e temos $\mathcal{U} \subset \bigcap_{n \in \mathbb{N}} A_{n}$.

Seja $x \in \bigcap_{n \in \mathbb{N}} A_{n}$. Dado $y \in U$ (aberto) $\subset Y$, existe $n_{0} \in \mathbb{N}$ tal que $y \in V_{n_{0}} \subset U$. Como $x \in A_{n_{0}}$, então existe $i \in I$ tal que $x \in T_{i}^{-1}\left(V_{n_{0}}\right)$, ou seja, $T_{i}(x) \in V_{n_{0}} \subset U$. Assim, $x$ é universal e temos $\bigcap_{n \in \mathbb{N}} A_{n} \subset \mathcal{U}$.

Portanto $\mathcal{U}$ é $G_{\delta}$.

É imediato então que $\mathcal{U}$ é residual (a) $\Leftrightarrow \mathcal{U}$ é denso (b).

(b) $\Rightarrow$ (c): Sejam $U \subset X$ e $V \subset Y$ abertos não-vazios.

Como $\mathcal{U}$ é denso, existe $x \in \mathcal{U} \cap U$. Então existe $i \in I$ tal que $T_{i}(x) \in V$ e temos $T_{i}(U) \cap V \neq \phi$.

$(\mathrm{c}) \Rightarrow(\mathrm{b}): \mathcal{U}$ é $G_{\delta}$. Como $X$ é espaço de Baire, basta mostrar que cada $A_{n}$ é denso em $X$.

Fixemos $n \in \mathbb{N}$. Dado $U$ (aberto não-vazio) $\subset X$, é possível obter $i \in I$ tal que $T_{i}(U) \cap V_{n} \neq \phi$, ou seja, existe $x \in U$ tal que $x \in T_{i}^{-1}\left(V_{n}\right) \subset A_{n}$. Assim $U \cap A_{n} \neq \phi$ e portanto $A_{n}$ é denso em $X$ (para cada $n \in \mathbb{N}$ ). 
Obs.: Como em muitas aplicações $X$ e $Y$ são espaços métricos, é comum termos na hipótese $X$ espaço métrico completo (portanto Baire) e $Y$ espaço métrico separável (portanto segundo-contável).

Uma explicação para o fato de haver poucos exemplos em que o conjunto dos elementos universais não é denso está no fato de que sob hipóteses razoavelmente naturais valem certos tipos de "Lei 0-1 Topológica", como as proposições seguintes, devidas a Peris e Grosse-Erdmann, respectivamente:

Proposição 1.3. (Lei 0-1) Sejam $X$ um espaço topológico e $T_{i}: X \rightarrow X$ aplicações contínuas. Suponha ainda que cada $T_{i}$ tenha imagem densa em $X$ e a familia $\left(T_{i}\right)_{i \in I}$ comute, ou seja, $T_{i} \circ T_{k}=T_{k} \circ T_{i}$ para todos $i, k \in I$.

Então o conjunto $\mathcal{U}$ dos elementos universais relativos à familia $\left(T_{i}\right)$ é OU vazio $O U$ denso (residual, se $X$ é um espaço de Baire segundo-contável).

Demonstração: [25]

Suponhamos que $\mathcal{U} \neq \phi$ e seja $x \in \mathcal{U}$. Para cada $i \in I$, seja $y_{i}=T_{i}(x)$. Temos: $\left\{y_{i}\right\}_{i \in I}$ é denso em $X$.

Fixemos $i \in I$.

Seja $U$ (aberto não-vazio) $\subset X$. Como $T_{i}$ tem imagem densa em $X$, então $T_{i}^{-1}(U)$ é aberto não-vazio.

Como $x$ é universal, existe $k \in I$ tal que $T_{k}(x) \in T_{i}^{-1}(U)$.

$\operatorname{Logo} T_{k}\left(y_{i}\right)=T_{k} \circ T_{i}(x)=T_{i} \circ T_{k}(x) \in U$ e temos $y_{i} \in \mathcal{U}$.

Assim $\mathcal{U} \supset\left\{y_{i}\right\}_{i \in I}$ (denso) e portanto $\mathcal{U}$ é denso em $X$.

A seguir temos outro tipo de "Lei 0-1 Topológica":

Proposição 1.4. (Lei 0-1) Sejam X um espaço topológico Hausdorff sem pontos isolados e $T_{i}: X \rightarrow X$ aplicações contínuas. Suponha ainda que, para cada $i \in I$, todo $T_{k}(k \in I)$, com no máximo um número finito de exceções, possa ser escrito na forma $T_{k}=T_{\lambda} \circ T_{i}$, para algum $\lambda \in I$.

Então o conjunto $\mathcal{U}$ dos elementos universais relativos à familia $\left(T_{i}\right)$ é OU vazio OU denso (residual, se $X$ é um espaço de Baire segundo-contável). 
Demonstração:

Suponhamos que $\mathcal{U} \neq \phi$ e seja $x \in \mathcal{U}$. Para cada $i \in I$, seja $y_{i}=T_{i}(x)$. Temos: $\left\{y_{i}\right\}_{i \in I}$ é denso em $X$.

Fixemos $i \in I$.

Dado $U$ (aberto não-vazio) $\subset X$, existem infinitos índices $k$ tais que $T_{k}(x) \in U$ (pois $X$ é Hausdorff e sem pontos isolados).

Com um número finito de exceções, todo $T_{k}$ pode ser escrito como $T_{k}=T_{\lambda} \circ T_{i}$, para algum $\lambda \in I$.

Podemos tomar então $k \in I$ tal que $T_{k}(x) \in U$ e $T_{k}=T_{\lambda} \circ T_{i}$ para algum $\lambda \in I$.

Então temos: $T_{\lambda}\left(y_{i}\right)=T_{\lambda} \circ T_{i}(x)=T_{k}(x) \in U$ e assim $y_{i}$ é universal.

Como $\mathcal{U} \supset\left\{y_{i}\right\}_{i \in I}$ (denso), segue que $\mathcal{U}$ é denso em $X$.

Exemplo: (em que $\mathcal{U}$ não é denso) [22]

Sejam $H$ um espaço de Hilbert, $\operatorname{dim} H=2,\{a, b\}$ base ortonormal de $H$ e $\left\{x_{n}\right\}_{n \in \mathbb{N}}$ (denso) $\subset H$.

Para cada $n \in \mathbb{N}$, tome $y_{n} \perp x_{n}$ com $\left\|y_{n}\right\|=n$.

Consideremos, para cada $n$, os operadores $T_{n}: H \rightarrow H$ dados por

$$
T_{n}(\alpha \cdot a+\beta \cdot b)=\alpha \cdot x_{n}+\beta \cdot y_{n} .
$$

Dado $\alpha \neq 0$, temos: $\left\{T_{n}(\alpha . a)\right\}_{n \in \mathbb{N}}=\left\{\alpha . x_{n}\right\}_{n \in \mathbb{N}}($ denso $) \subset H . \operatorname{Logo}[a] \backslash\{0\} \subset \mathcal{U}$.

Temos ainda: $\left\|T_{n}(\alpha \cdot a+\beta . b)\right\|^{2}=\left\|\alpha \cdot x_{n}+\beta \cdot y_{n}\right\|^{2}=\left\|\alpha \cdot x_{n}\right\|^{2}+\left\|\beta \cdot y_{n}\right\|^{2} \geq\left\|\beta \cdot y_{n}\right\|^{2}$.

Assim $\left\|T_{n}(\alpha . a+\beta . b)\right\| \geq\left\|\beta \cdot y_{n}\right\|=|\beta| . n \rightarrow \infty$ se $\beta \neq 0$.

Então $\alpha . a+\beta . b \notin \mathcal{U}$ se $\beta \neq 0$ e temos $\mathcal{U} \subset[a]$.

Portanto $\mathcal{U}=[a] \backslash\{0\}$ não é denso em $H$.

Os resultados anteriores sugerem a seguinte...

Definição 1.5. Sejam $X$ e $Y$ espaços topológicos. Uma família $\left(T_{i}\right)_{i \in I}$ de aplicações contínuas $T_{i}: X \rightarrow Y$ é chamada ... 
...DENSAMENTE UNIVERSAL se tem um conjunto denso de elementos universais;

...GENERICAMENTE UNIVERSAL se X é um espaço de Baire e a familia tem um conjunto residual de elementos universais.

Uma consequência imediata, mas importante, da residualidade é a existência de elementos universais comuns a diferentes famílias universais:

Proposição 1.6. Sejam $X$ um espaço de Baire fixado e $T_{i}^{(n)}: X \rightarrow Y_{n}\left(i \in I_{n}, n \in \mathbb{N}\right)$ aplicações contínuas.

Se cada família $\left(T_{i}^{(n)}\right)_{i \in I_{n}}(n \in \mathbb{N})$ é genericamente universal então existe um elemento em $X$ que é universal com respeito a cada uma destas familias. Mais ainda, o conjunto de elementos universais comuns é residual em $X$.

\section{Famílias universais em ambientes lineares}

Estaremos interessados em estudar famílias universais especificamente em ambientes lineares. Para tal (e para aplicarmos os resultados anteriores) vamos supor de agora em diante o seguinte:

$X$ é um espaço vetorial topológico (evt) de Baire ;

$Y$ é um evt metrizável e separável ;

$T_{n}: X \rightarrow Y \quad(n \in \mathbb{N})$ é uma família de transformações lineares contínuas.

Iniciaremos destacando uma importante condição suficiente, a qual futuramente irá dar origem ao chamado Critério de Hiperciclicidade.

Teorema 1.7. (Uma condição suficiente) Suponhamos que existam subconjuntos densos $X_{0} \subset X, Y_{0} \subset Y$ e aplicações (possivelmente descontínuas e não-lineares) $S_{n}: Y_{0} \rightarrow X$ tais que

(1) Para todo $x \in X_{0}, T_{n_{k}} x \rightarrow 0$ para alguma subsequência $\left(n_{k}\right)$;

(2) Para todo $y \in Y_{0},\left(S_{n} y\right)$ converge ; 
(3) Para todo $y \in Y_{0},\left(T_{n} \circ S_{n}\right) y \rightarrow y$.

Então, a sequência $\left(T_{n}\right)_{n \in \mathbb{N}}$ admite um conjunto $G_{\delta}$ denso, portanto residual, de vetores universais em $X$, relativos à familia $\left\{T_{n}\right\}_{n \in \mathbb{N}}$.

Demonstração: [25]

Sejam $U \subset X$ e $V \subset Y$ abertos não-vazios.

Tomemos $y \in Y_{0} \cap V$. Então $S_{n} y \rightarrow a \in X$.

$X_{0}$ (denso) $\subset X$ implica $X_{0}+a$ (denso) $\subset X$.

Logo, existe $u \in U \cap\left(X_{0}+a\right) \quad\left(u=x+a \in U\right.$, com $\left.x \in X_{0}\right)$.

De (1), existe uma subsequência $\left(n_{k}\right)$ tal que $T_{n_{k}}(u-a) \rightarrow 0$.

Seja então $\xi_{n_{k}}=S_{n_{k}}(y)+u-a \stackrel{k}{\rightarrow} u \in U$. Logo $\xi_{n_{k}} \in U$ para todo $k$ suf. gde..

Temos: $T_{n_{k}}\left(\xi_{n_{k}}\right)=T_{n_{k}} \circ S_{n_{k}}(y)+T_{n_{k}}(u-a) \rightarrow y \in V$. Logo $T_{n_{k}}\left(\xi_{n_{k}}\right) \in V$ para todo $k$ suf. gde..

Portanto existe $i \in \mathbb{N}$ tal que $T_{i}(U) \cap V \neq \phi$ e o resultado segue do Teorema 1.2.

Observações: (variações nas condições anteriores)

(a) É suficiente o atendimento das condições para uma determinada subsequência $\left(T_{m_{n}}\right)_{n} ;$

(b) Se todos os limites em (2) são 0, então podemos pedir apenas a convergência em (1);

De fato:

Sejam $U \subset X$ e $V \subset Y$ abertos não-vazios. Tomemos $x \in X_{0} \cap U$.

Então existe uma subsequência $\left(n_{k}\right)$ tal que $T_{n_{k}}(x) \rightarrow b \in Y$.

$Y_{0}$ (denso) $\subset Y$ implica $Y_{0}+b$ (denso) $\subset Y$.

Logo, existe $v \in V \cap\left(Y_{0}+b\right) \quad\left(v=y+b \in V\right.$, com $\left.y \in Y_{0}\right)$.

Seja então $\xi_{n_{k}}=x+S_{n_{k}}(v-b) \stackrel{k}{\rightarrow} x \in U$. Temos: $\xi_{n_{k}} \in U$ para todo $k$ suf. gde..

Também temos $T_{n_{k}}\left(\xi_{n_{k}}\right)=T_{n_{k}}(x)+T_{n_{k}} \circ S_{n_{k}}(v-b) \rightarrow b+v-b=v \in V$ e daí segue 
que $T_{n_{k}}\left(\xi_{n_{k}}\right) \in V$ para todo $k$ suf. gde..

Portanto existe $i \in \mathbb{N}$ tal que $T_{i}(U) \cap V \neq \phi$ e novamente pelo Teorema $1.2 \mathrm{o}$ resultado segue.

(c) A condição relativa "a uma subsequência" pode passar para (2) ou para (3).

Aplicação: No espaço $X=H(\mathbb{C})$ das funções inteiras em $\mathbb{C}$, com a topologia compactoaberta (da convergência uniforme sobre os compactos de $\mathbb{C}$ ), consideremos o subespaço $\mathcal{P}(\mathbb{C})$ dos polinômios sobre $\mathbb{C}$, o operador derivação $D: H(\mathbb{C}) \rightarrow H(\mathbb{C})$ e suas iteradas $D_{n}=D^{n} \quad \forall n \in \mathbb{N}$.

Sejam $X_{0}=Y_{0}=\mathcal{P}(\mathbb{C})$ (denso) $\subset H(\mathbb{C}), \quad S: Y_{0} \rightarrow X$ a aplicação dada por $S(f)(z)=\int_{0}^{z} f(\xi) d \xi$ e $S_{n}=S^{n} \quad \forall n \in \mathbb{N}$.

Temos então:

(1) $D_{n} f \rightarrow 0 \quad \forall f \in X_{0}=\mathcal{P}(\mathbb{C})$;

(2) $S_{n} g \rightarrow 0 \quad \forall g \in Y_{0}=\mathcal{P}(\mathbb{C})$;

(3) $\left(D_{n} \circ S_{n}\right) g=g \rightarrow g \quad \forall g \in Y_{0}$.

Do Teorema 1.7, segue então que $\left\{D, D^{2}, D^{3}, \ldots\right\}$ é uma família universal de operadores sobre $X=H(\mathbb{C})$ (exemplo de MacLane) com um conjunto residual de vetores universais.

Veremos agora que uma interessante e imediata consequência da residualidade é a possibilidade de escrever qualquer vetor do espaço $X$ como a soma de dois vetores universais:

Proposição 1.8. Se $\left(T_{n}\right)_{n \in \mathbb{N}}$ é uma familia genericamente universal em $X$, então cada vetor em $X$ é a soma de dois vetores universais, ou seja, $X=\mathcal{U}+\mathcal{U}$.

Demonstração:

$$
\mathcal{U} \supset \bigcap_{n \in \mathbb{N}} A_{n}\left(G_{\delta} \text { denso). Fixado } x \in X \text {, temos } x-\mathcal{U} \supset \bigcap_{n \in \mathbb{N}}\left(x-A_{n}\right) .\right.
$$


Logo $x-\mathcal{U}$ é residual.

Assim $(x-\mathcal{U}) \cap \mathcal{U} \neq \phi$ e portanto existem $u_{1}, u_{2} \in \mathcal{U}$ tais que $x-u_{1}=u_{2}$, isto é, $x=u_{1}+u_{2}$.

\section{Operadores hipercíclicos}

Neste trabalho iremos estudar um tipo particular de universalidade dentro do ambiente linear. Nos interessa o caso em que a família universal é constituída pelas iteradas de um único operador linear contínuo sobre um espaço vetorial topológico:

Definição 1.9. Sejam $X$ um evt e $T: X \rightarrow X$ um operador linear contínuo. Um vetor $x \in X$ é chamado HIPERCÍCLICO (para $T$ ) quando sua órbita sob $T$, dada por $\mathcal{O}(x ; T)=\left\{x, T x, T^{2} x, T^{3} x, \ldots\right\}$, é densa em $X$ (observe que neste caso o espaço $X$ é necessariamente separável).

O operador T é chamado HIPERCÍCLICO quando admite um vetor hipercíclico.

Denotaremos o conjunto de vetores hipercíclicos para $T$ por $\mathcal{H C}(T)$.

Conforme convenções adotadas anteriormente sobre famílias universais em ambientes lineares, bem como os tipos de espaços com os quais iremos trabalhar, vamos considerar de agora em diante o fenômeno da hiperciclicidade em espaços de Fréchet (espaços localmente convexos, metrizáveis e completos) separáveis.

O primeiro exemplo histórico de operador hipercíclico surgiu com a prova de Birkhoff em 1929 sobre a universalidade de translações sobre o espaço $H(\mathbb{C})$ da funções inteiras em $\mathbb{C}$ (com a topologia da convergência uniforme sobre os compactos de $\mathbb{C}$ ), a qual levou à conclusão de que, para todo $a \neq 0$ em $\mathbb{C}$, o operador dado por $T_{a}(f)(z)=f(z+a)$ sobre $H(\mathbb{C})$ é hipercíclico.

Outro exemplo importante de operador hipercíclico foi o operador de derivação sobre o mesmo espaço $H(\mathbb{C})$ das funções inteiras em $\mathbb{C}$ (MacLane - 1952). 
Os primeiros exemplos em espaços de Banach ou Hilbert foram os weighted backward shifts (Rolewicz - 1969).

Nestes últimos anos os operadores hipercíclicos têm sido estudados intensivamente.

Antes de passarmos aos resultados básicos sobre operadores hipercíclicos, convém tecer alguns comentários sobre a origem do termo "hipercíclico", a qual está associada a um problema famoso.

Começamos com a definição de vetor cíclico:

Definição 1.10. Seja $T$ um operador linear contínuo sobre um evt $E$. Um vetor $x \in E$ é dito CÍCLICO (para T) quando o espaço gerado por sua órbita sob $T$ é denso em $E$, ou seja, $\overline{[\mathcal{O}(x ; T)]}=E$.

Vetores cíclicos estão associados ao estudo de subespaços invariantes. Se um vetor $x \in E$ é cíclico (para $T$ ), então o único subespaço fechado e $T$-invariante que o contém é todo o espaço $E$.

Um problema famoso constitui-se na questão: Todo operador sobre um espaço de dimensão infinita sempre admite um subespaço fechado e invariante não-trivial ?

É imediato que um operador não admite nenhum subespaço fechado e invariante nãotrivial se, e somente se, todo vetor não-nulo é cíclico. O problema acima foi resolvido por Enflo em [19], onde foi construído um espaço de Banach que admite um operador onde todos os vetores não-nulos são cíclicos. O problema continua em aberto para espaços de Hilbert.

Da mesma forma que vetores cíclicos estão associados a subespaços invariantes, vetores hipercíclicos estão associados ao estudo de subconjuntos invariantes e o mesmo problema acima pode ser transportado naturalmente para este contexto. Entre ambas as noções, surgiu naturalmente a definição de vetores supercíclicos:

Definição 1.11. Seja $T$ um operador linear contínuo sobre um evt $E$. Um vetor $x \in E$ é dito SUPERCÍCLICO (para T) quando o conjunto $\left\{\alpha . T^{n} x ; n \in \mathbb{N}_{0}, \alpha\right.$ escalar $\}$ é denso em $E$.

A partir daí o termo "hipercíclico" se torna natural para a Definição 1.9 ! 
Destacaremos agora alguns resultados básicos sobre operadores hipercíclicos, os quais seguem imediatamente do que foi visto anteriormente sobre famílias universais em geral.

Iniciaremos observando que o fenômeno da hiperciclicidade é exclusivo para operadores sobre espaços de dimensão infinita:

Proposição 1.12. Seja $E$ um espaço de Fréchet. Se $\operatorname{dim} E<\infty$ então não existe nenhum operador hipercíclico sobre $E$.

A proposição acima é devida a Rolewicz [34]. Uma prova simples, consequência da investigação de formas canônicas para matrizes representantes de operadores sobre espaços de dimensão finita, e baseada numa idéia de Kitai [27] pode ser encontrada em [25].

O teorema seguinte é consequência do Teorema 1.2, combinado com a Proposição 1.3 ou com a Proposição 1.4:

Teorema 1.13. Sejam $E$ um espaço de Fréchet separável e $T: E \rightarrow E$ um operador linear contínuo sobre $E$. As seguintes afirmações são equivalentes:

(a) Té hipercíclico ;

(b) $T$ é genericamente hipercíclico (ou seja, $\mathcal{H C}(T)$ é residual em E);

(c) Dados abertos não-vazios $U$ e $V$ em $E$, é possivel obter $n_{0} \in \mathbb{N}_{0}$ com $T^{n_{0}}(U) \cap V \neq \phi$.

Caso as afirmações acima sejam verdadeiras, então o conjunto $\mathcal{H C}(T)$ dos vetores hipercíclicos é um subconjunto $G_{\delta}$ denso em $E$.

Corolário 1. Seja T um operador linear contínuo e invertível sobre um espaço de Fréchet separável E.

$T$ é hipercíclico se, e somente se, $T^{-1}$ é hipercíclico.

O teorema seguinte (Kitai, Gethner e Shapiro, Bès e Peris), consequência imediata do Teorema 1.7, constitui-se em uma poderosa ferramenta para se obter hiperciclicidade: 
Teorema 1.14. (O Critério de Hiperciclicidade) Sejam E um espaço de Fréchet separável e $T$ um operador linear contínuo sobre E. Suponhamos que existam subconjuntos densos $X_{0}$ e $Y_{0}$ de E, uma sequência crescente $\left(n_{k}\right)$ de naturais e aplicações (possivelmente não-lineares e descontínuas) $S_{n_{k}}: Y_{0} \rightarrow E$ tais que

(i) $\operatorname{para}$ cada $x \in X_{0}, T^{n_{k}} x \longrightarrow 0$,

(ii) para cada $y \in Y_{0}, S_{n_{k}} y \longrightarrow 0$,

(iii) para cada $y \in Y_{0},\left(T^{n_{k}} \circ S_{n_{k}}\right) y \longrightarrow y$.

(neste caso dizemos que T satisfaz o Critério para $\left(n_{k}\right)$ )

Então o operador $T$ é hipercíclico e o conjunto dos vetores hipercíclicos é subconjunto $G_{\delta}$ e denso de $E$ (portanto residual em $E$ ).

Aplicação: No espaço de Banach $E=\ell_{p}(1 \leq p<\infty)$ ou $E=c_{0}$, o operador linear contínuo $T: E \rightarrow E$ dado por $T\left(x_{1}, x_{2}, x_{3}, \ldots\right)=\left(a x_{2}, a x_{3}, a x_{4}, \ldots\right)$ (weighted backward shift), com $|a|>1$, é hipercíclico.

De fato: com $X_{0}=Y_{0}=c_{00}$ (denso) $\subset E, S\left(a_{1}, a_{2}, a_{3}, \ldots\right)=\frac{1}{a} \cdot\left(0, a_{1}, a_{2}, \ldots\right)$ e $S_{n}=S^{n}$ para todo $n \in \mathbb{N}$, temos:

(1) $T^{n} x \rightarrow 0 \quad \forall x \in X_{0}$;

(2) $S_{n} y \rightarrow 0 \quad \forall y \in Y_{0}$;

(3) $\left(T^{n} \circ S_{n}\right) y=y \rightarrow y \quad \forall y \in Y_{0}$.

Do Critério de Hiperciclicidade, segue que $T$ é um operador hipercíclico sobre $E$ (exemplo de Rolewicz).

O Critério de Hiperciclicidade (C.H.) possui uma forma mais fraca (essencialmente quando o operador satisfaz o Critério para toda a sequência dos naturais), hoje conhecida como Critério de Kitai. Para que se tenha uma idéia da força do C.H., até hoje não se tem um exemplo de um operador que seja hipercíclico mas não satisfaça o Critério em sua forma mais geral e já se tornou um problema clássico no estudo de operadores hipercíclicos determinar se o C.H. é ou não uma condição necessária para se ter hiperciclicidade, ou seja, questiona-se o quão perto ele está de uma caracterização dos operadores hipercíclicos. Condições equivalentes ao Critério foram obtidas por Bès e Peris [12] e Grivaux [24]. 
A seguir, duas proposições que são consequência (imediata) do fato de que se um operador é hipercíclico, então o conjunto dos vetores hipercíclicos é residual, ou seja, é um conjunto grande no sentido de categorias de Baire.

Chamamos uma atenção especial para a primeira delas, referente à existência de vetores hipercíclicos comuns a coleções enumeráveis de operadores hipercíclicos, o que dá origem a resultados surpreendentes e fascinantes.

Proposição 1.15. Seja $E$ um espaço de Fréchet separável. Se $T_{n}(n \in \mathbb{N})$ são operadores hipercíclicos sobre $E$, então existe um vetor em $E$ que é hipercíclico para todo $T_{n}$. Mais ainda, o conjunto de vetores hipercíclicos comuns é residual em E.

Proposição 1.16. Seja E um espaço de Fréchet separável. Se T é um operador hipercíclico sobre $E$ então todo vetor em $E$ pode ser escrito como soma de dois vetores hipercíclicos, ou seja, $E=\mathcal{H C}(T)+\mathcal{H C}(T)$.

Nota: Ressaltamos que a parte introdutória até aqui desenvolvida foi baseada no artigo "Universal families and hypercyclic operators"[25], de Karl-Goswin Grosse-Erdmann.

\section{Descrição do trabalho}

Antes de nos referirmos aos resultados do presente trabalho, vale a pena fazermos um resumo dos problemas típicos que costumam aparecer no estudo de operadores hipercíclicos:

- Obtenção de operadores hipercíclicos;

Este é, por natureza, o problema primário no estudo de operadores hipercíclicos. Além dos exemplos clássicos de Birkhoff [13], MacLane [28] e Rolewicz [34], destacamos os trabalhos de Gethner e Shapiro [21], Godefroy e Shapiro [22], Salas [35], Aron e Markose [5], dentre outros, além de alguns resultados gerais sobre existência de operadores hipercíclicos (Ansari [3] - Bonet e Peris [14], Bernal-González [8], Grivaux [24] [23]). 
- Existência de subespaços "grandes" de vetores hipercíclicos;

Dado um operador hipercíclico, seu conjunto de vetores hipercíclicos não é (mesmo incluindo o vetor nulo), em geral, um espaço vetorial. Desta forma é natural a busca de subespaços (de dimensão infinita, fechados e de dimensão infinita) onde todos os vetores à exceção do vetor nulo são hipercíclicos para o operador hipercíclico dado. Destacamos aqui os trabalhos de Godefroy e Shapiro [22], Herrero [26] - Bourdon [15] - Bès [11], Bernal-González e Montes-Rodríguez [10], Montes-Rodríguez [30], dentre outros.

- Existência de vetores hipercíclicos comuns a famílias não-enumeráveis de operadores hipercíclicos;

Sabemos da Proposição 1.15 que toda coleção enumerável de operadores hipercíclicos (sobre um mesmo espaço) admite vetores hipercíclicos comuns. Nada mais natural portanto do que questionar sobre a existência de vetores hipercíclicos comuns a todos os operadores de coleções não-enumeráveis de operadores hipercíclicos. Resultados nessa direção foram obtidos por Abakumov e Gordon [1], Bayart [7], Costakis e Sambarino [18].

- Construção de vetores hipercíclicos.

Em muitos casos os métodos utilizados para se obter hiperciclicidade são indiretos, ou melhor, não-construtivos. Como exemplo podemos citar a poderosa ferramenta que é o Critério de Hiperciclicidade, o qual garante hiperciclicidade sem construir vetores hipercíclicos. Assim sendo, a busca da construção de vetores hipercíclicos (mesmo que não sejam explícitos) se coloca como um problema de interesse no estudo de operadores hipercíclicos. Destacamos resultados obtidos por Aron e Markose [5], Aron, SeoaneSepúlveda e Weber [6], Seoane-Sepúlveda [36].

Neste trabalho estudamos essencialmente operadores hipercíclicos em espaços de funções inteiras, sob vários aspectos englobados pelos problemas típicos anteriormente mencionados.

No Capítulo 2 desenvolvemos um estudo relacionado (de certa forma) com dois dos problemas típicos acima descritos.

Costakis e Sambarino provaram em [18] que a coleção não-enumerável de todas as translações não-nulas (como chamaremos de agora em diante as translações por um com- 
plexo diferente de zero) em $H(\mathbb{C})$ (com a topologia compacto-aberta) admite vetores (neste caso funções) hipercíclicos comuns a todos os operadores da coleção.

É sabido, a partir de resultados de Herrero [26], Bourdon [15] e Bès [11], que todo operador hipercíclico sobre um espaço de Fréchet admite a existência de um subespaço denso (portanto de dimensão infinita) e invariante no qual todos os vetores (à exceção do vetor nulo) são hipercíclicos para o operador em questão. Embora esses resultados se refiram a cada operador hipercíclico isoladamente e não a coleções de operadores hipercíclicos, é possível (utilizando um argumento similar a Herrero-Bourdon-Bès) obter um subespaço hipercíclico comum, denso, de dimensão enumerável (infinita), para coleções de operadores que comutam dois a dois, desde que exista pelo menos um vetor hipercíclico comum a todos os operadores da coleção considerada, como é o caso da coleção de translações estudada por Costakis e Sambarino, por exemplo.

Nesse segundo capítulo nós mostramos, por um outro argumento, que o conjunto de vetores hipercíclicos comuns a todas a translações não-nulas em $H(\mathbb{C})$ contém (a menos do vetor nulo, é claro) um espaço vetorial de dimensão infinita (denso) e, mais ainda, a dimensão desse espaço é c (cardinalidade do contínuo).

No terceiro capítulo trabalhamos com a obtenção de uma coleção de operadores hipercíclicos sobre $H(\mathbb{C})$, com certas características especiais.

Um importante teorema de Godefroy e Shapiro em [22] estabelece que todo operador de convolução (linear, contínuo e que comuta com as translações) sobre $H\left(\mathbb{C}^{n}\right)$ e que não seja múltiplo da identidade é necessariamente hipercíclico. A partir desse resultado, tornou-se natural a busca de operadores hipercíclicos e que não sejam de convolução, sobre $H\left(\mathbb{C}^{n}\right)$.

Em [5], Aron e Markose introduziram uma coleção de operadores sobre $H(\mathbb{C})$ dados por $T(f)(z)=f^{\prime}(\lambda z+b),|\lambda| \geq 1, \lambda, b \in \mathbb{C}$, os quais são hipercíclicos e não são de convolução se $\lambda \neq 1$. Porém a demonstração apresentada em [5] contém uma falha no caso $b \neq 0$.

Este problema é resolvido no Capítulo 3, onde é apresentada uma demonstração que também estabelece o caso $b \neq 0$, "completando" de certa forma o exemplo original de Aron e Markose.

No quarto capítulo tecemos algumas considerações sobre o tamanho do conjunto de vetores hipercíclicos de determinados operadores investigando uma noção semelhante ao que se conhece como "porosidade". 
Se $T$ é um operador hipercíclico sobre um espaço de Fréchet $E$ temos, a partir do Teorema 1.13 , que o conjunto $\mathcal{H C}(T)$ (dos vetores hipercíclicos relativos a $T$ ) é residual em $E$ (ou seja, contém um conjunto $G_{\delta}$ denso em $E$ ). Isto significa que tal conjunto é grande no sentido topológico (de categorias de Baire) ou, equivalentemente, seu complementar é "magro" (conjunto de $1^{a}$ categoria, isto é, união enumerável de conjuntos cujo fecho tem interior vazio).

Melhorar este resultado significa mostrar que o conjunto de vetores hipercíclicos atende a uma propriedade que englobe a residualidade ou, equivalentemente, que seu complementar possui alguma propriedade mais restritiva do que ser de $1^{a}$ categoria. O conceito de $\sigma$-porosidade se apresenta como uma alternativa quando buscamos algo mais restritivo do que ser de $1^{a}$ categoria.

Em [16], Brunkalla resgatou o conceito de porosidade em espaços métricos com o objetivo de estudar o tamanho dos conjuntos de vetores cíclicos dos weighted backward shifts nos espaços $\ell_{p}$.

Como nos interessa prioritariamente pesquisar operadores hipercíclicos sobre espaços de funções inteiras, procuramos então desenvolver neste trabalho um estudo semelhante ao de Brunkalla, porém voltado particularmente para as translações em $H(\mathbb{C})$. Para tal, necessitamos inicialmente de uma noção de porosidade que funcione bem ao trabalharmos com as normas que geram a topologia compacto-aberta em $H(\mathbb{C})$, sem perder as principais características do conceito original.

Desta forma, introduzimos nesse Capítulo 4 o conceito de $t$-porosidade em $H(\mathbb{C})$, similar (em certo sentido) ao conceito de porosidade utilizado por Brunkalla. Provamos que todo conjunto $\sigma$-t-poroso é de $1^{a}$ categoria e mostramos finalmente que o complementar do conjunto dos vetores hipercíclicos para cada translação em $H(\mathbb{C})$ é $\sigma$-t-poroso.

No Capítulo 5 estendemos resultados referentes à construção de funções hipercíclicas para translações e à existência de funções hipercíclicas comuns a coleções não-enumeráveis de translações, ambos em $H(\mathbb{C})$, para outros espaços (bem mais gerais) de funções inteiras.

Um teorema de Runge (Teorema 5.1) garante que se uma função é analítica numa vizinhança de um conjunto compacto $(\mathrm{em} \mathbb{C}$ ) cujo complementar é conexo, então essa função pode ser uniformemente aproximada por polinômios nesse compacto. Tal resultado tem se mostrado como a melhor ferramenta (até agora conhecida) para a obtenção de outros relativos à hiperciclicidade de translações em $H(\mathbb{C})$. Para ilustrar: Aron e Markose, 
utilizando Runge, construíram em [5] funções hipercíclicas para translações em $H(\mathbb{C})$, apresentando assim uma prova mais simples para o exemplo de Birkhoff. Também utilizando fortemente o Teorema de Runge, Costakis e Sambarino provaram em [18] que a coleção (não-enumerável) de todas as translações não-nulas em $H(\mathbb{C})$ admite funções hipercíclicas comuns.

Nesse quinto capítulo nós inicialmente adaptamos o Teorema de Runge para que tenhamos um resultado que funcione sobre certos domínios (mesmo não-compactos) em qualquer espaço de Banach. A partir daí, estendemos os resultados acima referidos, de Aron e Markose e de Costakis e Sambarino, para subespaços do espaço $H_{b}(E)$ das funções inteiras de tipo limitado (em alguns casos para todo o espaço $H_{b}(E)$ ), podendo $E$ pertencer a uma vasta classe de espaços de Banach. 


\section{Capítulo 2}

\section{Uma observação sobre "lineabilidade"}

Conjuntos de vetores hipercíclicos não são (mesmo incluindo o vetor nulo), em geral, espaços vetoriais. A partir desta constatação torna-se interessante investigarmos se tais conjuntos contêm ou não subespaços vetoriais de dimensão infinita ou, mais ainda, subespaços vetoriais fechados de dimensão infinita dos espaços onde estão inseridos. Tais propriedades são conhecidas respectivamente como "lineabilidade"(da palavra "lineability") e "espaçabilidade"(de "spaceability"), conforme as definições abaixo.

Definição 2.1. Diremos que um subconjunto $G$ de um espaço vetorial topológico $E$ é...

...LINEÁ VEL quando o conjunto $G \cup\{0\}$ contém um subespaço vetorial de dimensão infinita de $E$.

...ESPAÇÁVEL quando o conjunto $G \cup\{0\}$ contém um subespaço vetorial fechado e de dimensão infinita de $E$.

Quanto à espaçabilidade (mais difícil), podemos apenas citar alguns exemplos. Em [10], Bernal-González e Montes-Rodríguez concluem que o conjunto de vetores hipercíclicos de cada translação não-nula sobre $H(\mathbb{C})$ (exemplo de Birkhoff) é espaçável. Por outro lado, Montes-Rodríguez provou em [30] que os "weighted backward shifts" (exemplo de Rolewicz) não comportam subespaços fechados de dimensão infinita cujos vetores não- 
nulos sejam todos hipercíclicos.

A questão da lineabilidade já está resolvida se considerarmos cada operador hipercíclico isoladamente. Dado um operador hipercíclico sobre um espaço de Fréchet, existe um subespaço denso (portanto de dimensão infinita) e invariante no qual todos os vetores não-nulos são hipercíclicos para o operador em questão (Herrero [26] - Bourdon [15] - Bès [11]).

Se existir um vetor hipercíclico comum a uma coleção de operadores hipercíclicos que comutam dois a dois, um argumento baseado no resultado de Herrero-Bourdon-Bès prova a existência de um subespaço hipercíclico (a menos do vetor nulo), denso, de dimensão enumerável e infinita (por ser denso), comum a todos os operadores da família considerada (observação de Nilson Bernardes).

Nos interessa aqui investigar, através de um outro argumento, a lineabilidade do conjunto de vetores hipercíclicos comuns a uma certa coleção de operadores hipercíclicos. Nosso ponto de partida é um (surpreendente) resultado que destaca essa coleção e o conjunto que queremos mostrar ser lineável.

Teorema 2.2. (Costakis e Sambarino, [18]) Consideremos o espaço $H(\mathbb{C})$ das funções inteiras em $\mathbb{C}$, com a topologia compacto-aberta. Se a $\in \mathbb{C}$ e $\tau_{a}: H(\mathbb{C}) \rightarrow H(\mathbb{C})$ é o operador de translação por a $\left(\tau_{a}(f)(z)=f(z+a)\right)$, então o conjunto $G=\bigcap_{a \neq 0} \mathcal{H C}\left(\tau_{a}\right)$ não é vazio, ou seja, existem funções em $H(\mathbb{C})$ que são hipercíclicas para todo $\tau_{a}$ ( $a \neq 0$ em $\mathbb{C})$.

$\mathrm{Na}$ verdade, Costakis e Sambarino mostraram mais ainda: que esse conjunto $S$ das funções inteiras hipercíclicas comuns a todas as translações não-nulas em $H(\mathbb{C})$ é residual.

Neste segundo capítulo mostramos (através de uma prova simples) que o conjunto $G=\bigcap_{a \neq 0} \mathcal{H C}\left(\tau_{a}\right)$ é lineável, obtendo um espaço denso de dimensão não-enumerável contido (a menos do vetor nulo) em $G$. Para tal, utilizaremos fortemente um resultado que já se tornou clássico no estudo de operadores hipercíclicos sobre espaços de funções inteiras:

Teorema 2.3. (Godefroy e Shapiro, [22]) Se $T: H\left(\mathbb{C}^{n}\right) \rightarrow H\left(\mathbb{C}^{n}\right)$ é um operador de convolução (operador linear contínuo que comuta com todas as translações em $H\left(\mathbb{C}^{n}\right)$ ) e $T$ não é múltiplo da identidade, então $T$ é hipercíclico. 
Corolário 1. Todo operador de convolução não-nulo sobre $H\left(\mathbb{C}^{n}\right)$ possui imagem densa.

O lema seguinte é parte de um teorema mais geral em [9], onde Bernal-González busca caracterizar (sob certas condições) operadores que preservam a universalidade, isto é, operadores que levam elementos universais em elementos universais. Tendo em vista o que pretendemos provar, segue uma versão adaptada ao nosso contexto.

Lema 2.4. Seja $T: E \rightarrow E$ um operador hipercíclico. Se $S: E \rightarrow E$ é um operador linear contínuo que comuta com $T$ e sua imagem $S(E)$ é densa em $E$, então $S(\mathcal{H C}(T)) \subset \mathcal{H C}(T)$, ou seja, o conjunto $\mathcal{H C}(T)$ dos vetores hipercíclicos relativos ao operador $T$ é S-invariante.

Demonstração:

Seja $x \in \mathcal{H C}(T)\left(\mathcal{O}(x ; T)=\left\{x, T x, T^{2} x, T^{3} x, \ldots\right\}\right.$, é denso em $\left.E\right)$.

Como $S$ comuta com $T$, então

$$
\mathcal{O}(S x ; T)=\left\{S x, T S x, T^{2} S x, \ldots\right\}=\left\{S x, S T x, S T^{2} x, \ldots\right\}=S(\mathcal{O}(x ; T))
$$

Como $\mathcal{O}(x ; T)$ é denso em $E, S$ é contínuo e tem imagem $S(E)$ densa, podemos concluir que $S(\mathcal{O}(x ; T))$ é denso em $E$ e portanto $S x \in \mathcal{H C}(T)$.

Agora, com todas as ferramentas em mãos, podemos finalmente estabelecer o resultado anunciado anteriormente:

Proposição 2.5. O espaço $H(\mathbb{C})$ (Fréchet, com a topologia compacto-aberta) admite um subespaço de dimensão infinita no qual todos os vetores (neste caso funções), com exceção do vetor nulo, são funções hipercíclicas comuns a todas as translações não-nulas sobre $H(\mathbb{C})$, ou seja, o conjunto $G=\bigcap_{a \neq 0} \mathcal{H C}\left(\tau_{a}\right)$ é lineável.

Demonstração:

Do Teorema 2.2, sabemos que o conjunto $G=\bigcap_{a \neq 0} \mathcal{H C}\left(\tau_{a}\right)$ não é vazio e podemos tomar portanto $f \in G$. Consideremos o seguinte subespaço de $H(\mathbb{C})$ :

$$
M=\left[\tau_{b} f\right]_{b \in \mathbb{C}} \quad \text { (espaço gerado pelo conjunto }\left\{\tau_{b} f ; b \in \mathbb{C}\right\} \text { ). }
$$


Iremos mostrar que $M$ tem dimensão infinita e $M \backslash\{0\} \subset G$.

Comecemos mostrando que toda função não-nula em $M$ é hipercíclica para todas as translações não-nulas em $H(\mathbb{C})$.

Seja $g \in M \backslash\{0\}$. Então $g=z_{1} \tau_{b_{1}} f+\ldots+z_{k} \tau_{b_{k}} f=\left(z_{1} \tau_{b_{1}}+\ldots+z_{k} \tau_{b_{k}}\right) f$, com $g \neq 0$ e $z_{1}, \ldots, z_{k}, b_{1}, \ldots, b_{k} \in \mathbb{C}$.

Fixemos $a \neq 0$ em $\mathbb{C}$.

O operador $S: H(\mathbb{C}) \rightarrow H(\mathbb{C})$ dado por $S=z_{1} \tau_{b_{1}}+\ldots+z_{k} \tau_{b_{k}}$ é claramente um operador de convolução. E não-nulo, pois $g \neq 0$ e $g=S f$.

Logo, segue do Corolário 1 do Teorema 2.3 que $S$ tem imagem densa em $H(\mathbb{C})$.

Agora, como $S$ é um operador linear contínuo que comuta com $\tau_{a}$ (de fato, comuta com todas as translações), $S$ tem imagem densa e $f \in \mathcal{H C}\left(\tau_{a}\right)$, podemos concluir pelo Lema 2.4 que $g=S f \in \mathcal{H C}\left(\tau_{a}\right)$.

Portanto $g \in G$ e temos $M \backslash\{0\} \subset G$.

A título de observação apenas, notemos que $M$ é $\tau_{a}$-invariante, para todo $a \in \mathbb{C}$.

Para ver que $M$ tem dimensão infinita e concluir a demonstração, é suficiente constatar que $M$ contém as órbitas de $f$ sob todas as translações. Como $f \in G$, então cada órbita de $f$ sob cada translação não-nula é densa em $H(\mathbb{C})$. Portanto $M$ é denso e necessariamente tem dimensão infinita. Apesar deste ser o caminho mais simples e rápido para finalizar a prova, não deixa de ser interessante mostrarmos que $M$ tem dimensão infinita através da independência linear do conjunto (não-enumerável) $\left\{\tau_{b} f ; b \in \mathbb{C}\right\}$, que gera $M$.

Seja $z_{1} \tau_{b_{1}} f+\ldots+z_{n} \tau_{b_{n}} f=0, \operatorname{com} z_{1}, \ldots, z_{n}, b_{1}, \ldots, b_{n} \in \mathbb{C}$ e $b_{i} \neq b_{j}$ se $i \neq j$.

Então $\left(z_{1} \tau_{b_{1}}+\ldots+z_{n} \tau_{b_{n}}\right) f=0$. Como 0 (função nula) nunca é hipercíclica, $f$ é hipercíclica para as translações e $S=z_{1} \tau_{b_{1}}+\ldots+z_{k} \tau_{b_{k}}$ é um operador de convolução sobre $H(\mathbb{C})$, segue do Lema 2.4 e do Corolário 1 do Teorema 2.3 que $\left(z_{1} \tau_{b_{1}}+\ldots+z_{k} \tau_{b_{k}}\right)=0$ (operador nulo sobre $H(\mathbb{C})$ ) necessariamente.

A partir daí podemos concluir que $z_{1}=z_{2}=\ldots=z_{n}=0$, usando polinômios interpoladores.

De fato, para cada $i=1, \ldots, n$ podemos tomar um polinômio $p_{i} \in H(\mathbb{C})$ tal que $p_{i}\left(b_{i}\right)=1$ e $p_{i}\left(b_{j}\right)=0$ para todo $j \neq i$. Então $0=\left(z_{1} \tau_{b_{1}}+\ldots+z_{n} \tau_{b_{n}}\right)\left(p_{i}\right)(0)=$ 
$z_{1} \tau_{b_{1}}\left(p_{i}\right)(0)+\ldots+z_{n} \tau_{b_{n}}\left(p_{i}\right)(0)=z_{1} p_{i}\left(b_{1}\right)+\ldots+z_{n} p_{i}\left(b_{n}\right)=z_{i}$ para cada $i=1, \ldots, n$.

Assim, o conjunto $\left\{\tau_{b} f ; b \in \mathbb{C}\right\}$ é linearmente independente e portanto $M$ tem dimensão (infinita) $\mathfrak{c}$ (cardinalidade do contínuo).

Segue que o conjunto $G=\bigcap_{a \neq 0} \mathcal{H C}\left(\tau_{a}\right)$ é lineável. 


\section{Capítulo 3}

\section{Operadores hipercíclicos que não são de convolução}

Já vimos (e utilizamos) no capítulo anterior um importante resultado (Teorema 2.3) no estudo de operadores hipercíclicos sobre espaços de funções inteiras. Godefroy e Shapiro [22] estabeleceram que se considerarmos o espaço de Fréchet $H\left(\mathbb{C}^{n}\right)$, das funções analíticas em $\mathbb{C}^{n}$ com a topologia da convergência uniforme sobre os conjuntos compactos de $\mathbb{C}^{n}$, então todo operador de convolução (linear, contínuo e que comuta com as translações) que não seja múltiplo da identidade é necessariamente hipercíclico.

Uma vez que este resultado de Godefroy e Shapiro generalizou outros até então conhecidos, como a hiperciclicidade das translações (exemplo de Birkhoff) e da derivação (exemplo de MacLane) em $H(\mathbb{C})$, tornou-se relevante a procura por operadores hipercíclicos que não sejam de convolução, sobre esses espaços $H\left(\mathbb{C}^{n}\right), n \geq 1$.

A família de operadores sobre $H(\mathbb{C})$ dados por $T(f)(z)=f^{\prime}(\lambda z+b)$, com $|\lambda| \geq 1$, $\lambda, b \in \mathbb{C}$, foi introduzida por Aron e Markose em [5] como exemplo de coleção de operadores hipercíclicos sobre $H(\mathbb{C})$, os quais não são de convolução se $\lambda \neq 1$.

A prova de Aron e Markose em [5] se baseia numa forma mais fraca do Critério de Hiperciclicidade (C.H., Teorema 1.14) conhecida como Critério de Kitai. Como na maioria das aplicações do Critério, os autores usam o espaço $Z=\mathcal{P}(\mathbb{C})($ dos polinômios sobre $\mathbb{C}$ ) 
como subconjunto denso em $H(\mathbb{C})$ e buscam uma aplicação $S: Z \rightarrow Z$ que satisfaça as seguintes condições:

(1) $T^{n} f \rightarrow 0$ para toda $f \in Z$;

(2) $S^{n} f \rightarrow 0$ para toda $f \in Z$;

(3) $\left(T^{n} \circ S^{n}\right) f \rightarrow f$ para toda $f \in Z$.

Apesar do resultado (hiperciclicidade dos operadores) estar correto, a prova em [5] apresenta uma falha, no seguinte sentido: as expressões para $S\left(z^{k}\right)$ (e $\left.S^{n}\left(z^{k}\right)\right)$ não são corretas para a verificação das condições (do C.H.) acima nos casos em que $b \neq 0$.

Resolvemos este problema neste terceiro capítulo. É apresentada uma demonstração que também estabelece a hiperciclicidade para o caso $b \neq 0$.

Antes de apresentarmos nossa demonstração, recordemos que no C.H. não é necessário considerar as iteradas de uma mesma aplicação $S: Z \rightarrow Z$. Se temos um subconjunto denso $Z \subset H(\mathbb{C})$ e uma sequência de aplicações $S_{n}: Z \rightarrow H(\mathbb{C})$ tais que (1) acima é satisfeita e também

(2') $S_{n} f \rightarrow 0$ para toda $f \in Z$;

$\left(3^{\prime}\right)\left(T^{n} \circ S_{n}\right) f \rightarrow f$ para toda $f \in Z$

são satisfeitas, então o Critério "funciona" da mesma forma, ou seja, $T$ é hipercíclico (na verdade, para a forma mais geral do C.H., estas condições precisam ser satisfeitas apenas para uma subsequência $\left(n_{k}\right)$ de índices).

Estabelecemos de forma "completa", a seguir, o resultado originalmente apresentado por Aron e Markose:

Teorema 3.1. Definamos o operador $T: H(\mathbb{C}) \rightarrow H(\mathbb{C})$ dado por $T(f)(z)=f^{\prime}(\lambda z+b)$, com $\lambda, b \in \mathbb{C}$. Se $|\lambda| \geq 1$, então $T$ é hipercíclico.

Demonstração:

Iremos aplicar o C.H. com $Z=\mathcal{P}(\mathbb{C})$ (conjunto dos polinômios sobre $\mathbb{C}$ ), que é denso em $H(\mathbb{C})$. 
Visando obter uma expressão razoável para cada aplicação $S_{n}: Z \rightarrow H(\mathbb{C})$, observemos inicialmente que

$$
T=R_{\lambda} \circ \tau_{b} \circ D
$$

sendo $R_{\lambda}, \tau_{b}$ e $D$ os operadores sobre $H(\mathbb{C})$ dados por

$$
\begin{aligned}
& R_{\lambda}(f)(z)=f(\lambda z), \\
& \tau_{b}(f)(z)=f(z+b) \quad(\text { translação por } b), \\
& D(f)(z)=f^{\prime}(z) \text { (derivação). }
\end{aligned}
$$

De fato, temos, para todos $f \in H(\mathbb{C})$ e $z \in \mathbb{C}$ :

$$
\begin{aligned}
\left(R_{\lambda} \circ \tau_{b} \circ D\right)(f)(z) & =R_{\lambda}\left(\tau_{b}(D f)\right)(z)= \\
& =\tau_{b}(D f)(\lambda z)= \\
& =D f(\lambda z+b)= \\
& =f^{\prime}(\lambda z+b)= \\
& =T(f)(z) \quad \therefore \quad T=R_{\lambda} \circ \tau_{b} \circ D .
\end{aligned}
$$

Note que se $\lambda \neq 1$, então $R_{\lambda}$ não comuta com $\tau_{a}(a \neq 0)$ ou $D$ e temos $D \circ R_{\lambda}=\lambda\left(R_{\lambda} \circ D\right)$ e $\tau_{a} \circ R_{\lambda}=R_{\lambda} \circ \tau_{\lambda a}$. Logo $T$ não comuta com todas as translações se $\lambda \neq 1\left(\tau_{a} \circ T=T \circ \tau_{\lambda a}\right)$ e portanto $T$ não é um operador de convolução neste caso.

Com estas relações, é fácil computar

$$
T^{n}=\lambda^{\frac{n(n-1)}{2}} R_{\lambda^{n}} \circ \tau_{-r_{n}} \circ D^{n} \quad \text {, onde } \quad r_{n}=-b \cdot \sum_{l=0}^{n-1} \lambda^{l}
$$

É fácil ver através da expressão acima que a condição (1) no C.H. é satisfeita.

Como uma motivação, observemos que a condição $\left(3^{\prime}\right)$ no C.H. nos fornece uma dica para a obtenção das aplicações $S_{n}$. A terceira condição no C.H. nos diz que as aplicações $S_{n}: Z \rightarrow H(\mathbb{C})$ devem se comportar (assintoticamente, quando $n \rightarrow \infty$ ) como inversas à direita sobre $Z$ para os operadores $T^{n}$. 
Assim, se denotarmos por $A D$ (anti-derivação) o "operador formal" definido apenas no conjunto $A=\{1\} \cup\left\{d(z+c)^{k} ; k \in \mathbb{N}, c, d \in \mathbb{C}\right\}$ por

$$
\begin{aligned}
& A D\left[d(z+c)^{k}\right]=\frac{d(z+c)^{k+1}}{k+1} \quad k \in \mathbb{N}, c, d \in \mathbb{C} \\
& A D[1]=(z-b)
\end{aligned}
$$

e como $D \circ A D=I d_{A}$, a terceira condição do C.H. torna natural tentarmos (no conjunto $\left.1, z, z^{2}, \ldots, z^{k}, \ldots\right)$

$$
S=A D \circ \tau_{-b} \circ R_{1 / \lambda} \quad \text { e } \quad S_{n}=S^{n} \quad \text { para todo } n \in \mathbb{N} \text {. }
$$

Observemos que $A D$ é apenas uma "ferramenta operacional", cuja utilidade é a de nos guiar a expressões bem definidas e explícitas das aplicações $S_{n}: Z \rightarrow H(\mathbb{C})$ na base $\left\{1, z, z^{2}, \ldots, z^{k}, \ldots\right\}$ de $Z=\mathcal{P}(\mathbb{C})$.

Chegamos então a

$$
S_{n}\left(z^{k}\right)=\frac{k !}{(k+n) ! \lambda^{k n} \lambda^{\frac{n(n-1)}{2}}}\left(z+r_{n}\right)^{k+n} \quad\left(k \in \mathbb{N}_{0}, n \in \mathbb{N}\right)
$$

e estendemos linearmente a $Z$.

Como esperado, esta construção funciona bem para a verificação da condição $\left(3^{\prime}\right)$, mas enfrentamos problemas com as potências elevadas de $\lambda$ em $\left(z+r_{n}\right)^{k+n}$ quando tentamos verificar $\left(2^{\prime}\right)$.

Contudo, se considerarmos

$$
S_{n}\left(z^{k}\right)=\frac{k !}{(k+n) ! \lambda^{k n} \lambda^{\frac{n(n-1)}{2}}}\left[\left(z+r_{n}\right)^{k+n}+\Delta_{n, k}(z)\right] \quad n \in \mathbb{N}, k \in \mathbb{N}_{0}
$$

onde $\Delta_{n, k}(z)$ é qualquer polinômio em $z$ com grau menor ou igual a $n-1$, é fácil ver que ainda temos $\left(3^{\prime}\right)$ satisfeita (veja a expressão para $T^{n}$ ).

Portanto a idéia agora será usar $\Delta_{n, k}(z)$ apropriados para eliminar as potências elevadas de $\lambda$ no binômio $\left(z+r_{n}\right)^{k+n}$ (e podemos perfeitamente fazer isso, pois as 
potências elevadas de $\lambda$ vêm associadas a potências baixas de $z$ na expansão binomial).

Fixando então

$$
\Delta_{n, k}(z)=-\sum_{j=k+1}^{k+n}\left(\begin{array}{c}
k+n \\
j
\end{array}\right) z^{k+n-j} r_{n}^{j} \quad n \in \mathbb{N}, k \in \mathbb{N}_{0},
$$

teremos finalmente

$$
\begin{aligned}
S_{n}\left(z^{k}\right) & =\frac{k !}{(k+n) ! \lambda^{k n} \lambda^{\frac{n(n-1)}{2}}}\left[\left(z+r_{n}\right)^{k+n}+\Delta_{n, k}(z)\right]= \\
& =\frac{k !}{(k+n) ! \lambda^{k n} \lambda^{\frac{n(n-1)}{2}}}\left[\sum_{j=0}^{k}\left(\begin{array}{c}
k+n \\
j
\end{array}\right) z^{k+n-j} r_{n}^{j}\right],
\end{aligned}
$$

aplicações as quais estendemos linearmente a $Z$.

Como o grau de cada polinômio $\Delta_{n, k}(z)$ é $n-1$, ( $\left.3^{\prime}\right)$ é satisfeita e agora, se considerarmos $|z| \leq R$ e fixarmos $k \in \mathbb{N}_{0}$, temos então, para cada $j=0,1, \ldots, k$

$$
\begin{gathered}
\left|\frac{k !}{(k+n) ! \lambda^{k n} \lambda^{\frac{n(n-1)}{2}}}\left(\begin{array}{c}
k+n \\
j
\end{array}\right) z^{k+n-j} r_{n}^{j}\right| \leq \frac{k !(k+n) ! R^{k+n-j}\left|r_{n}\right|^{j}}{(k+n) !|\lambda|^{k n}|\lambda|^{\frac{n(n-1)}{2}}(k+n-j) ! j !} \leq \\
\leq \frac{k ! R^{k+n-j}|b|^{j} n^{j}|\lambda|^{j n}}{|\lambda|^{k n}|\lambda|^{\frac{n(n-1)}{2}}(k+n-j) ! j !} \stackrel{n}{\longrightarrow} 0 \quad(\operatorname{se}|\lambda| \geq 1) .
\end{gathered}
$$

Portanto $S_{n}\left(z^{k}\right) \stackrel{n}{\rightarrow} 0,\left(2^{\prime}\right)$ é finalmente satisfeita e podemos concluir (pelo C.H.) que o operador $T$ é hipercíclico.

A título de observação, convém mencionar que esta parte (Capítulo 3) constitui um trabalho conjunto com Gustavo Fernández, realizado em 2004 na Kent State University e publicado no Journal of Mathematical Analysis and Applications. [20] 


\section{Capítulo 4}

\section{Sobre porosidade e hiperciclicidade}

É bem conhecido (Teorema 1.13) que o conjunto $\mathcal{H C}(T)$ de vetores hipercíclicos de um operador hipercíclico $T: E \rightarrow E$ (Fréchet) é "topologicamente grande", ou melhor, é residual em $E$ (contém um conjunto $G_{\delta}$ denso em $E$ ).

Podemos melhorar (de alguma forma) o resultado acima?

Observemos que um conjunto (em um espaço de Baire) é residual se, e somente se, seu complementar é um conjunto de $1^{a}$ categoria (união enumerável de conjuntos cujo fecho tem interior vazio). Desta forma, se olharmos para o conjunto $\mathcal{N H C}(T)=E \backslash \mathcal{H C}(T)$, uma alternativa que se apresenta para a questão acima é a busca de uma noção de conjuntos "topologicamente pequenos" que seja mais forte do que a noção de $1^{a}$ categoria e a partir daí tentar provar que $\mathcal{N H C}(T)$ é pequeno.

A definição abaixo pode ajudar em nossa busca:

Definição 4.1. Um subconjunto $A$ de um espaço métrico $(X, d)$ é chamado POROSO quando existe uma constante $c>0$ (chamada "uma constante de porosidade" para $O$ conjunto A) tal que, para cada $x \in A$ e cada $R \in(0,1)$, existe $y \in X$ com $B[y ; c R] \subset$ $B[x ; R]$ e $B[y ; c R] \cap A=\phi$.

Um conjunto $\sigma-P O R O S O$ é uma união enumerável de conjuntos porosos. 
Obs.: Podemos dizer, a grosso modo, que um conjunto poroso possui a seguinte propriedade: arbitrariamente próximos a qualquer ponto do conjunto existem "buracos"(conjuntos abertos de seu complementar), sendo que esses buracos são proporcionais ao tamanho das vizinhanças que estamos considerando para a noção de proximidade. Este modo de ver os conjuntos porosos pode ser útil futuramente neste capítulo.

Exemplos: Seguem alguns exemplos básicos sobre porosidade.

(a) Seja $(X, d)$ um espaço métrico tal que, dados $x \in X$ e $0<\alpha<\beta<1$, é possível obter $y \in X \operatorname{com} \alpha<d(x, y)<\beta$ (por exemplo, $X$ pode ser um espaço normado).

Se $A \subset X$ é um conjunto tal que inf $\{d(x, y) ; x \neq y$ em $A\} \geq r>0$, então $A$ é poroso e $c=\min \{r / 4,1 / 4\}$ é uma constante de porosidade para $A$.

De fato, dados $x \in A$ e $R \in(0,1)$, temos:

(i) Se $R<r$, tome $y \in X$ tal que $R / 4<d(x, y)<R / 2$.

Se $w \in B[y ; c R]$, temos: $d(w, y) \leq c R \leq R / 4$ e portanto $w \neq x$. Além disso:

$d(w, x) \leq d(w, y)+d(y, x) \leq c R+\frac{R}{2} \leq \frac{r R}{4}+\frac{r}{2}<\frac{r}{4}+\frac{r}{2}<r . \operatorname{Logo} B[y ; c R] \cap A=\phi$.

$d(w, x) \leq c R+\frac{R}{2} \leq \frac{R}{4}+\frac{R}{2}<R$. Então $B[y ; c R] \subset B[x ; R]$.

(ii) Se $R \geq r$, tome $y \in X$ tal que $r / 4<d(x, y)<r / 2$.

Se $w \in B[y ; c R]$, temos: $d(w, y) \leq c R \leq \frac{r R}{4}<\frac{r}{4}$ e portanto $w \neq x$. Temos ainda:

$d(w, x) \leq d(w, y)+d(y, x) \leq c R+\frac{r}{2} \leq \frac{r R}{4}+\frac{r}{2}<\frac{r}{4}+\frac{r}{2}<r . \log 0 B[y ; c R] \cap A=\phi$.

$d(w, x) \leq c R+\frac{r}{2} \leq \frac{R}{4}+\frac{R}{2}<R$. Então $B[y ; c R] \subset B[x ; R]$.

De qualquer modo existe $y \in X$ tal que $B[y ; c R] \subset B[x ; R]$ e $B[y ; c R] \cap A=\phi$ e portanto segue que $A$ é poroso.

Em particular: qualquer conjunto finito em $X$ é poroso; $A=\mathbb{N} \times \mathbb{N} \subset \mathbb{R}^{2}$ (métrica euclidiana) é poroso; $A=\left\{\left(x_{1}, x_{2}, \ldots\right) ; x_{i} \in\{0,1\}, i=1,2, \ldots\right\} \subset \ell_{\infty}$ (métrica usual) é poroso.

(b) Com alguns cálculos, é possível mostrar que $V=\{ \pm 1 / n ; n \in \mathbb{N}\} \subset \mathbb{R}$ (métrica usual) não é poroso. [16] 
(c) Por outro lado, é fácil ver que $V^{\prime}=\{( \pm 1 / n, 0) ; n \in \mathbb{N}\} \subset \mathbb{R}^{2}$ (métrica euclidiana) é poroso.

Para finalizarmos esta parte introdutória, frisamos que todo conjunto $\sigma$-poroso é de $1^{a}$ categoria, mas a recíproca não é verdadeira. Iremos omitir neste ponto a prova de que todo conjunto $\sigma$-poroso é de $1^{a}$ categoria, pois teremos mais à frente neste capítulo uma prova bem semelhante, com técnica parecida, apesar de em outro contexto (ver Corolário 1 da Proposição 4.5). Quanto à recíproca, é sabido que conjuntos $\sigma$-porosos em $\mathbb{R}$ (métrica usual) têm medida nula ([16]) e desta forma podemos providenciar um contra-exemplo: basta tomar um conjunto de $1^{a}$ categoria e que não tenha medida nula (ver [32], pág. 4) e este conjunto não pode ser portanto $\sigma$-poroso.

Estas considerações mostram que a noção de $\sigma$-porosidade é mais forte do que a noção de $1^{a}$ categoria, no sentido em que se torna mais restritiva quando tentamos definir "conjuntos pequenos". Portanto, uma das primeiras idéias naturais a partir deste fato é investigar o tamanho dos conjuntos de vetores que não são hipercíclicos para os operadores hipercíclicos clássicos, conjuntos esses que sabemos ser de $1^{a}$ categoria. Seriam eles $\sigma$-porosos?

Brunkalla iniciou esse tipo de estudo em [16], voltado para os weighted backward shifts $B_{\lambda}: \ell_{p} \rightarrow \ell_{p}(1 \leq p<\infty)$, dados por $B_{\lambda}\left(x_{1}, x_{2}, x_{3}, \ldots\right)=\left(\lambda x_{2}, \lambda x_{3}, \lambda x_{4}, \ldots\right)$ (operadores hipercíclicos, quando $|\lambda|>1$ ).

Neste capítulo nos propomos a realizar um estudo semelhante (relacionando hiperciclicidade e porosidade) com as translações em $H(\mathbb{C})$. Porém, antes de desenvolvermos tal estudo, vale a pena considerarmos inicialmente o caso dos weighted backward shifts, tendo em vista alguns elementos comuns.

É ainda um problema em aberto determinar se o conjunto $\mathcal{N H C}\left(B_{\lambda}\right)=\ell_{p} \backslash \mathcal{H C}\left(B_{\lambda}\right)$ dos vetores que não são hipercíclicos para os weighted backward shifts $B_{\lambda}(|\lambda|>1)$ é ou não $\sigma$-poroso. Até agora, o melhor resultado que temos se refere ao conjunto $\mathcal{N} \mathcal{S C}\left(B_{\lambda}\right)$ dos vetores que não são supercíclicos.

Recordemos que um operador linear contínuo $T$ sobre um espaço de Fréchet $E$ é supercíclico quando existe um vetor $x \in E$ tal que o conjunto $\left\{\alpha T^{n}(x) ; \alpha \in \mathbb{K}, n \in \mathbb{N}_{0}\right\}$ é denso em $E$ (neste caso o vetor $x$ é dito um vetor supercíclico para o operador $T$ ). 
Segue uma prova completa do resultado de Brunkalla sobre a $\sigma$-porosidade do conjunto $\mathcal{N S C}\left(B_{\lambda}\right)$ dos vetores que não são supercíclicos para os weighted backward shifts:

Teorema 4.2. Para cada $\lambda \neq 0$, o conjunto $\mathcal{N S C}\left(B_{\lambda}\right)$ dos vetores que não são supercíclicos para o weighted backward shift $B_{\lambda}$ sobre $\ell_{p}(1 \leq p<\infty)$ é $\sigma$-poroso.

Demonstração:

Observemos inicialmente que para cada $\lambda \neq 0$ o weighted backward shift $B_{\lambda}$ é um operador supercíclico e todos esses $B_{\lambda}$ compartilham o mesmo conjunto de vetores supercíclicos (imediato a partir da definição).

Assim, iremos considerar, sem perda de generalidade, apenas o caso $B_{1}$ (backward shift).

Seja $\left\{x^{(k)}=\left(x_{1}^{(k)}, x_{2}^{(k)}, \ldots\right)\right\}_{k \in \mathbb{N}}$ uma sequência densa de vetores não nulos em $\ell_{p}$.

Para cada $k, l \in \mathbb{N}$, definamos $A_{k, l}=\left\{x \in \ell_{p} ; \operatorname{dist}\left(x^{(k)}, \mathbb{K O}\left(x ; B_{1}\right)\right)>\frac{1}{l}\right\}$, sendo $\mathbb{K} \mathcal{O}\left(x ; B_{1}\right)=\left\{\alpha B_{1}^{n}(x) ; \alpha \in \mathbb{K}, n \in \mathbb{N}_{0}\right\}$.

É imediato que $\bigcup_{k, l} A_{k, l} \subset \mathcal{N S C}\left(B_{1}\right)$.

Consideremos agora $x \in \mathcal{N S C}\left(B_{1}\right)$.

Existem $w \in \ell_{p}$ e $\epsilon>0$ tais que $B(w ; \epsilon) \cap \mathbb{K} \mathcal{O}\left(x ; B_{1}\right)=\phi$.

Como $\left\{x^{(k)}\right\}_{k}$ é denso em $\ell_{p}$, existe $k_{0} \in \mathbb{N}$ tal que $x^{\left(k_{0}\right)} \in B(w ; \epsilon / 2)$.

Tomemos $l_{0} \in \mathbb{N}$ tal que $\frac{1}{l_{0}}<\frac{\epsilon}{2}$.

Temos: $\operatorname{dist}\left(x^{\left(k_{0}\right)}, \mathbb{K} \mathcal{O}\left(x ; B_{1}\right)\right) \geq \frac{\epsilon}{2}>\frac{1}{l_{0}}$ e daí $x \in A_{k_{o}, l_{o}} \subset \bigcup_{k, l} A_{k, l}$.

Portanto $\mathcal{N} \mathcal{S C}\left(B_{1}\right)=\bigcup_{k, l} A_{k, l}$.

Fixemos agora $k, l \in \mathbb{N}$. Queremos mostrar que $A_{k, l}$ é poroso.

Sejam dados $x=\left(x_{1}, x_{2}, x_{3}, \ldots\right) \in A_{k, l}$ e $R \in(0,1)$.

Escolha $n \in \mathbb{N}$ tal que $x^{\prime}=\left(x_{1}, \ldots, x_{n}, 0,0, \ldots\right)$ satisfaz $\left\|x-x^{\prime}\right\|<R / 3$ e seja 


$$
y=\left(x_{1}, x_{2}, \ldots, x_{n}, \frac{R}{3\left\|x^{(k)}\right\|} x_{1}^{(k)}, \frac{R}{3\left\|x^{(k)}\right\|} x_{2}^{(k)}, \ldots\right) .
$$

Note que $B_{1}^{n} y=\frac{R}{3\left\|x^{(k)}\right\|} x^{(k)}$. Então $x^{(k)} \in \mathbb{K} \mathcal{O}\left(y ; B_{1}\right)$ e temos $y \notin A_{k, l}$.

Agora: $\|y-x\| \leq\left\|y-x^{\prime}\right\|+\left\|x^{\prime}-x\right\|<\frac{R}{3\left\|x^{(k)}\right\|}\left\|x^{(k)}\right\|+\frac{R}{3}<\frac{2 R}{3}$.

Consideremos finalmente $c=\min \left\{\frac{1}{3 l}, \frac{1}{3 l\left\|x^{(k)}\right\|}\right\}$ (note que $c=c(k, l)$ ).

Se $z \in B[y ; c R]$, então $\|z-y\| \leq R / 3 l \leq R / 3$.

Logo $\|z-x\| \leq R$ e temos $B[y ; c R] \subset B[x ; R]$.

Mais uma vez $z \in B[y ; c R]$ implica $\left\|\frac{3\left\|x^{(k)}\right\|}{R} B_{1}^{n} z-x^{(k)}\right\|=$

$=\left\|\frac{3\left\|x^{(k)}\right\|}{R} B_{1}^{n} z-\frac{3\left\|x^{(k)}\right\|}{R} B_{1}^{n} y\right\| \leq \frac{3\left\|x^{(k)}\right\|}{R}\|z-y\| \leq \frac{1}{l}$.

Então $z \notin A_{k, l}, B[y ; c R] \cap A_{k, l}=\phi$ e podemos concluir que $A_{k, l}$ é poroso.

Portanto $\mathcal{N S C}\left(B_{1}\right)=\bigcup_{k, l} A_{k, l}$ é $\sigma$-poroso.

Queremos agora estudar as relações entre porosidade e hiperciclicidade, considerando outros operadores hipercíclicos clássicos, a saber, as translações sobre $H(\mathbb{C})$.

Mas, neste caso, não seria produtivo tentar usar uma expressão explícita para uma métrica em $H(\mathbb{C})$ com a definição até agora conhecida de porosidade, uma vez que nós raramente trabalhamos com uma métrica explícita que gere a topologia compacto-aberta em $H(\mathbb{C})$.

É muito mais natural o trabalho com as (semi-)normas $\left(p_{n}\right)_{n}$ que definem a topologia localmente convexa (metrizável e completa) da convergência uniforme nos conjuntos compactos de $\mathbb{C}$, dadas por $p_{n}(f)=\sup _{|z| \leq n}|f(z)|, n \in \mathbb{N}$. 
Desta forma, iremos definir em $H(\mathbb{C})$ uma noção de porosidade relacionada com as semi-normas que geram sua topologia. Essa deve ser uma definição "menos métrica", mas naturalmente ligada à topologia considerada.

É claro que precisamos manter alguns elementos em comum com a definição anterior de porosidade (veja a observação após a Definição 4.1):

Definição 4.3. Um conjunto $A \subset H(\mathbb{C})$ será chamado t-POROSO se, e somente se, existir uma constante $c>0$ (chamada "uma constante de t-porosidade" para o conjunto A) tal que, para cada $f \in A$, cada $n \in \mathbb{N}$ e cada $R \in(0,1)$, existem $g \in H(\mathbb{C})$ e $n^{\prime} \in \mathbb{N}$ com $p_{n^{\prime}}(h-g) \leq c R$ implicando $p_{n}(h-f) \leq R$ e $h \notin A$.

Um conjunto $\sigma$-t-POROSO é uma união enumerável de conjuntos t-porosos.

Obs.: A notação que introduzimos abaixo será utilizada adiante:

$$
\begin{aligned}
& B_{n}[f ; R]=\left\{g \in H(\mathbb{C}) ; p_{n}(g-f) \leq R\right\} ; \\
& B_{n}(f ; R)=\left\{g \in H(\mathbb{C}) ; p_{n}(g-f)<R\right\} .
\end{aligned}
$$

$\mathrm{O}$ primeiro resultado interessante relacionado com este novo conceito de $t$-porosidade diz que $\sigma$ - $t$-porosidade implica em $1^{a}$ categoria:

Lema 4.4. Se $F \subset H(\mathbb{C})$ é um conjunto fechado e t-poroso, então $F$ é "raro"("nowhere dense”), ou seja, $\operatorname{int}(\bar{F})=\phi$.

Demonstração:

Queremos mostrar que $\operatorname{int} \bar{F}=\phi$, mas como $F=\bar{F}$, então resta mostrar apenas que o conjunto aberto $A=H(\mathbb{C}) \backslash F$ é denso em $H(\mathbb{C})$.

Mas isto é imediato uma vez que temos $F t$-poroso. De fato, como os conjuntos $B_{n}[f ; R](n \in \mathbb{N}, R>0)$ formam bases de vizinhanças de funções $f \in F$, temos diretamente da definição de conjunto $t$-poroso que tais vizinhanças sempre contêm elementos de $H(\mathbb{C}) \backslash F$ e daí o resultado segue.

Proposição 4.5. Se $A \subset H(\mathbb{C})$ é t-poroso, então $A$ é raro. 
Demonstração:

Como qualquer subconjunto de um conjunto raro é também raro, precisamos apenas mostrar que se $A \subset H(\mathbb{C})$ é $t$-poroso então seu fecho $\bar{A}$ é $t$-poroso (o resultado seguirá do Lema 4.4).

Seja $A \subset H(\mathbb{C})$ um conjunto $t$-poroso, com uma constante de $t$-porosidade $c>0$.

Queremos mostrar que $\bar{A}$ é $t$-poroso.

Sejam dados $f \in \bar{A}, n \in \mathbb{N}$ e $R \in(0,1)$.

Se $f \in A$ então existem $g \in H(\mathbb{C})$ e $n^{\prime} \in \mathbb{N}$ com $B_{n^{\prime}}[g ; c R] \subset B_{n}[f ; R]$ e $B_{n^{\prime}}[g ; c R] \cap A=\phi$ (veja a Definição 4.3 com a notação da observação seguinte).

É claro que $B_{n^{\prime}}[g ; c R / 2] \subset B_{n}[f ; R]$ e $B_{n^{\prime}}[g ; c R / 2] \cap \bar{A}=\phi$.

De fato, se $h \in B_{n^{\prime}}[g ; c R / 2] \cap \bar{A}$, então existe uma $u \in B_{n^{\prime}}[h ; c R / 2] \cap A$, o que implicaria $p_{n^{\prime}}(u-g) \leq c R$, isto é, $u \in B_{n^{\prime}}[g ; c R] \cap A$ (absurdo).

Se $f \in \bar{A} \backslash A$, então existe $\tilde{f} \in B_{n}[f ; R / 2] \cap A$.

Temos $B_{n}[\tilde{f} ; R / 2] \subset B_{n}[f ; R]$.

Da mesma forma que antes (caso $f \in A$ ), é claro que podemos tomar $g \in H(\mathbb{C})$ e $n^{\prime} \in \mathbb{N}$ tais que $B_{n^{\prime}}[g ; c R / 4] \subset B_{n}[\tilde{f} ; R / 2] \subset B_{n}[f ; R]$ e $B_{n^{\prime}}[g ; c R / 4] \cap \bar{A}=\phi$.

Portanto a constante $c / 4$ serve para a $t$-porosidade de $\bar{A}$.

Corolário 1. Se $A \subset H(\mathbb{C})$ é $\sigma$-t-poroso, então $A$ é um conjunto de $1^{a}$ categoria.

Finalmente, provaremos que o conjunto dos vetores que não são hipercíclicos para cada translação sobre $H(\mathbb{C})$ é $\sigma$-t-poroso (já é conhecido que tais conjuntos são de $1^{a}$ categoria).

Vamos considerar, sem perda de generalidade, a translação $\tau_{1}: H(\mathbb{C}) \rightarrow H(\mathbb{C})$ dada por $\tau_{1}(f)(z)=f(z+1)$.

Teorema 4.6. O conjunto $\mathcal{N H C}\left(\tau_{1}\right)$ dos vetores que não são hipercíclicos para a translação $\tau_{1}$ sobre $H(\mathbb{C})$ é $\sigma$-t-poroso.

Demonstração: 
Seja $\left\{f_{k}\right\}_{k \in \mathbb{N}}$ um conjunto denso em $H(\mathbb{C})$.

Para cada $(m, k, l) \in \mathbb{N} \times \mathbb{N} \times \mathbb{N}$, definamos

$$
A_{m, k, l}=\left\{f \in H(\mathbb{C}) ; p_{m}\left(f_{k}-\tau_{1}^{j} f\right)>\frac{1}{l} \quad \text { para todo } j \in \mathbb{N}_{0}\right\} \text {. }
$$

É claro que $\mathcal{N H C}\left(\tau_{1}\right)=\bigcup_{m, k, l} A_{m, k, l}$.

De fato:

Se $f \in A_{m, k, l}, \operatorname{com} m, k, l \in \mathbb{N}$, então a órbita de $f$ por $\tau_{1}$ não intersecta $B_{m}\left[f_{k} ; \frac{1}{l}\right]$ (vizinhança de $f_{k}$ ) e portanto $f$ não é um vetor hipercíclico para $\tau_{1}$.

Por outro lado, dada $f \in \mathcal{N} \mathcal{H C}\left(\tau_{1}\right)$, existem $g \in H(\mathbb{C}), m \in \mathbb{N}$ e $\epsilon>0$ tais que $p_{m}\left(g-\tau_{1}^{j} f\right)>\epsilon$ para todo $j \in \mathbb{N}_{0}$, pois $f$ não é um vetor hipercíclico para $\tau_{1}$.

Tomemos $k \in \mathbb{N}$ tal que $p_{m}\left(f_{k}-g\right)<\frac{\epsilon}{2}$ e $l \in \mathbb{N}$ tal que $\frac{1}{l}<\frac{\epsilon}{2}$.

Suponhamos que exista $j_{0} \in \mathbb{N}$ tal que $p_{m}\left(f_{k}-\tau_{1}^{j_{0}} f\right)<\frac{\epsilon}{2}$.

Então $p_{m}\left(g-\tau_{1}^{j_{0}} f\right) \leq p_{m}\left(g-f_{k}\right)+p_{m}\left(f_{k}-\tau_{1}^{j_{0}} f\right)<\epsilon$ (contradição).

Logo, obrigatoriamente, $p_{m}\left(f_{k}-\tau_{1}^{j} f\right) \geq \frac{\epsilon}{2}>\frac{1}{l}$ para todo $j \in \mathbb{N}_{0}$, ou seja, $f \in A_{m, k, l}$.

Uma vez estabelecido que $\mathcal{N} \mathcal{H C}\left(\tau_{1}\right)=\bigcup_{m, k, l} A_{m, k, l}$, iremos provar que cada $A_{m, k, l}$ é $t$-poroso. Para isso, fixemos $m, k, l \in \mathbb{N}$.

Sejam $f \in A_{m, k, l}, n \in \mathbb{N}, R \in(0,1)$.

Escolhamos $j_{0} \in \mathbb{N}$ tal que $j_{0}>m+n$ e consideremos $B=\tau_{1}^{j_{0}}(\{|z| \leq m\})=$ $\{|z| \leq m\}+j_{0}$ (translação por $j_{0}$ do conjunto $\left.\{|z| \leq m\}\right)$.

Então $B \cap\{|z| \leq n\}=\phi$.

Considere agora $\epsilon=\min \left\{\frac{2 R}{3}, \frac{1}{2 l}\right\}>0$.

Pelo Teorema de Runge, existe $g \in H(\mathbb{C})$ (até mesmo um polinômio) tal que:

$$
\sup _{|z| \leq n}|g(z)-f(z)|<\epsilon \leq \frac{2 R}{3} \quad \text { e }
$$




$$
\sup _{z \in B}\left|g(z)-\tau_{1}^{-j_{0}} f_{k}(z)\right|<\epsilon \leq \frac{1}{2 l} .
$$

Temos então $g \in H(\mathbb{C})$ tal que

$$
p_{m}\left(\tau_{1}^{j_{0}} g-f_{k}\right)=\sup _{B}\left|g(z)-\tau_{1}^{-j_{0}} f_{k}(z)\right|<\epsilon \leq \frac{1}{2 l}<\frac{1}{l} \quad \therefore g \notin A_{m, k, l} .
$$

Tome agora $n^{\prime}=j_{0}+m \in \mathbb{N}$ e fixe $c=\frac{1}{3 \cdot l}>0$.

Se $h \in H(\mathbb{C})$ satisfaz $p_{n^{\prime}}(h-g) \leq c R$, temos:

$$
\begin{aligned}
& p_{n}(h-f) \leq p_{n}(h-g)+p_{n}(g-f) \leq p_{n^{\prime}}(h-g)+p_{n}(g-f) \leq \frac{R}{3 \cdot l}+\frac{2 R}{3} \leq R \quad \mathrm{e} \\
& p_{m}\left(\tau_{1}^{j_{0}} h-f_{k}\right) \leq p_{m}\left(\tau_{1}^{j_{0}} h-\tau_{1}^{j_{0}} g\right)+p_{m}\left(\tau_{1}^{j_{0}} g-f_{k}\right)< \\
& <\sup _{|z| \leq m}\left|\tau_{1}^{j_{0}}(h-g)\right|+\frac{1}{2 l}=\sup _{B}|h-g|+\frac{1}{2 l} \leq \sup _{|z| \leq j_{0}+m}|h-g|+\frac{1}{2 l} \leq \\
& \leq p_{n^{\prime}}(h-g)+\frac{1}{2 l} \leq \frac{R}{3 \cdot l}+\frac{1}{2 l}<\frac{1}{2 l}+\frac{1}{2 l} \quad \therefore \quad h \notin A_{m, k, l} .
\end{aligned}
$$

Desta forma temos:

$$
p_{n^{\prime}}(h-g) \leq c R \Rightarrow p_{n}(h-f) \leq R \text { e } h \notin A_{m, k, l} .
$$

Portanto, $A_{m, k, l}$ é $t$-poroso, para todos $m, k, l \in \mathbb{N}$ e finalmente podemos concluir que $\mathcal{N H C}\left(\tau_{1}\right)=\bigcup_{m, k, l} A_{m, k, l}$ é $\sigma$-t-poroso. 


\section{Capítulo 5}

\section{Hiperciclicidade para translações através do Teorema de Runge}

No estudo de hiperciclicidade para translações em $H(\mathbb{C})$, uma ferramenta em particular tem se mostrado como a mais apropriada, a saber:

Teorema 5.1. (Runge) Se $f$ é holomorfa em uma vizinhança de um conjunto compacto $K \subset \mathbb{C}$ e $\mathbb{C} \backslash K$ é conexo, então $f$ pode ser uniformemente aproximada por polinômios em $K$. ([17], p.85)

O Teorema de Runge se torna uma ferramenta natural quando lidamos com hiperciclicidade de translações não apenas porque estamos considerando a topologia compacto-aberta em $H(\mathbb{C}$ ) (ou seja, a topologia da convergência uniforme sobre os conjuntos compactos de $\mathbb{C}$ ), ou porque cada função inteira é o limite de uma série polinomial, mas talvez principalmente porque podemos produzir típicos conjuntos compactos como no Teorema de Runge (uniões disjuntas de bolas, por exemplo) com translações de bolas.

Apresentamos aqui alguns exemplos para ilustrar este fato: Em [5], Aron e Markose fornecem uma prova mais simples do exemplo de Birkhoff utilizando o Teorema de Runge; Em [18], Costakis e Sambarino, também utilizando fortemente o Teorema de Runge, provam que existe um conjunto residual de funções hipercíclicas comuns a todas as translações $\tau_{a}, a \neq 0$, em $H(\mathbb{C})$. 
Em ambos os casos anteriormente descritos o conjunto compacto $K$ usado no Teorema de Runge pode ser considerado como uma união finita de bolas fechadas disjuntas, portanto um tipo particularmente regular (e simples) de conjunto compacto em $\mathbb{C}$. Desta forma, é natural questionarmos se temos resultados similares (ao de Runge) para conjuntos mais simples em outros espaços e o que podemos produzir com tais resultados no que se refere à hiperciclicidade de translações.

De fato, neste capítulo nós mostramos que é possível produzir esse tipo de estudo. Obtemos uma adaptação do Teorema de Runge de modo que este novo resultado possa ser utilizado com certos domínios não-compactos, porém em qualquer espaço de Banach, o que permitirá a extensão de teoremas clássicos sobre translações em $H(\mathbb{C})$ para outros espaços de funções inteiras.

Iniciamos estabelecendo o principal resultado do capítulo.

Teorema 5.2. Sejam $B_{1}$ e $B_{2}$ duas bolas fechadas disjuntas em um espaço de Banach $E$. Se $f$ é uma função holomorfa e limitada numa vizinhança uniforme de $B_{1} \cup B_{2}$ (por vizinhança uniforme de um conjunto $A$ nós queremos dizer $A+B(0, \delta)$, para algum $\delta>0$ ), então $f$ pode ser uniformemente aproximada por polinômios em $B_{1} \cup B_{2}$.

\section{Demonstração:}

Seja dado $\epsilon>0$.

Inicialmente, como $f$ é holomorfa e limitada numa vizinhança uniforme de $B_{1} \cup B_{2}$, podemos aproximar $f$ uniformemente por polinômios $p_{1}$ em $B_{1}$ e $p_{2}$ em $B_{2}$, isto é, existem $p_{1}, p_{2} \in \mathcal{P}(E)$ tais que $\sup _{x \in B_{j}}\left|p_{j}(x)-f(x)\right|<\epsilon / 2, j=1,2$.

De fato, como existe $\delta>0$ tal que $f$ é holomorfa e limitada em $\left(B_{1} \cup B_{2}\right)+B(0, \delta)$, então o raio de limitação de $f$ no centro de cada bola é estritamente maior do que o raio da bola. Mas como o raio de convergência (em cada centro) é maior ou igual ao raio de limitação ([31], p.53), a série de Taylor de $f$ em torno de cada centro irá convergir uniformemente na bola correspondente. Portanto podemos tomar $p_{1}$ e $p_{2}$ como polinômios de Taylor.

Seja $M=\sup _{x \in B_{1} \cup B_{2}}\left\{\left|p_{1}(x)\right|+\left|p_{2}(x)\right|\right\}$.

Pelo Teorema de separação de Hahn-Banach, existem $\gamma, \gamma^{\prime} \in \mathbb{R}$ e $\varphi \in E^{\prime}$ tais que 
$\operatorname{Re} \varphi(a)<\gamma^{\prime}<\gamma \leq \operatorname{Re} \varphi(b)$, sempre que $a \in B_{1}$ e $b \in B_{2}$.

Para vermos isto, consideremos $c_{1}$ e $c_{2}$ como os centros e $r_{1}$ e $r_{2}$ como os raios das bolas $B_{1}$ e $B_{2}$, respectivamente.

Vamos distinguir dois casos:

$1^{\circ}$ caso) Uma das bolas está centrada na origem:

Suponhamos, sem perda de generalidade, que $c_{1}=0$.

Como as bolas são disjuntas, temos $\left\|c_{2}\right\|-r_{2}>r_{1}$. Podemos tomar então $r^{\prime} \in \mathbb{R}$ tal que $r_{1}<r^{\prime}<\left\|c_{2}\right\|-r_{2}$ e considerar $B^{\prime}=B\left(0 ; r^{\prime}\right)$.

Temos $B_{1} \subset B^{\prime}$ (aberto) e $B_{2} \cap B^{\prime}=\phi$.

Pelo Teorema de separação de Eidelheit (Hahn-Banach geométrico) - ver 2.2.26 em [29], existem $\varphi \in E^{\prime}$ e $\lambda \in \mathbb{R}$ tais que

$$
\operatorname{Re} \varphi\left(a^{\prime}\right)<\lambda \leq \operatorname{Re} \varphi(b) \quad a^{\prime} \in B^{\prime}, b \in B_{2} .
$$

Note que, para todo $a^{\prime} \in B^{\prime}$, temos $-\operatorname{Re} \varphi\left(a^{\prime}\right)=\operatorname{Re} \varphi\left(-a^{\prime}\right)$, com $-a^{\prime} \in B^{\prime}$.

Logo $\lambda>\left|\operatorname{Re} \varphi\left(a^{\prime}\right)\right|$ para todo $a^{\prime} \in B^{\prime}$ e assim $\lambda>0$.

Agora, dado $a \neq 0$ em $B_{1}$, temos $a=\frac{2 \cdot\|a\|}{r_{1}+r^{\prime}} \cdot a^{\prime}$, com $a^{\prime}=\frac{a}{\|a\|} \cdot \frac{r_{1}+r^{\prime}}{2} \in B^{\prime}$.

Temos então $\operatorname{Re} \varphi(a)=\frac{2 \cdot\|a\|}{r_{1}+r^{\prime}} \cdot \operatorname{Re} \varphi\left(a^{\prime}\right)<\frac{2 \cdot\|a\|}{r_{1}+r^{\prime}} \cdot \lambda \leq \frac{2 \cdot r_{1}}{r_{1}+r^{\prime}} \cdot \lambda<\lambda$.

Pondo $\lambda^{\prime}=\frac{2 \cdot r_{1}}{r_{1}+r^{\prime}} \cdot \lambda$, temos: $\operatorname{Re} \varphi(a)<\lambda^{\prime}<\lambda \leq \operatorname{Re} \varphi(b), a \in B_{1}, b \in B_{2}$.

$2^{o}$ caso) Caso geral:

Seja $T: E \rightarrow E$ dada por $T(x)=x-c_{1}$. Então $T\left(B_{1}\right)$ e $T\left(B_{2}\right)$ são bolas fechadas e disjuntas, sendo $T\left(B_{1}\right)$ centrada na origem.

Pelo estudo feito no caso anterior, existem $\varphi \in E^{\prime}$ e $\lambda^{\prime}, \lambda \in \mathbb{R}$ tais que

$$
\operatorname{Re} \varphi(T(a))<\lambda^{\prime}<\lambda \leq \operatorname{Re} \varphi(T(b)) \quad a \in B_{1}, b \in B_{2} .
$$

$\Uparrow$

$$
\operatorname{Re} \varphi\left(a-c_{1}\right)<\lambda^{\prime}<\lambda \leq \operatorname{Re} \varphi\left(b-c_{1}\right) \quad a \in B_{1}, b \in B_{2} .
$$


$\Uparrow$

$\operatorname{Re} \varphi(a)-\operatorname{Re} \varphi\left(c_{1}\right)<\lambda^{\prime}<\lambda \leq \operatorname{Re} \varphi(b)-\operatorname{Re} \varphi\left(c_{1}\right) \quad a \in B_{1}, b \in B_{2}$.

$\Uparrow$

$\operatorname{Re} \varphi(a)<\lambda^{\prime}+\operatorname{Re} \varphi\left(c_{1}\right)<\lambda+\operatorname{Re} \varphi\left(c_{1}\right) \leq \operatorname{Re} \varphi(b) \quad a \in B_{1}, b \in B_{2}$.

Pondo $\gamma^{\prime}=\lambda^{\prime}+\operatorname{Re} \varphi\left(c_{1}\right)$ e $\gamma=\lambda+\operatorname{Re} \varphi\left(c_{1}\right)$, temos finalmente

$$
\operatorname{Re} \varphi(a)<\gamma^{\prime}<\gamma \leq \operatorname{Re} \varphi(b) \quad a \in B_{1}, b \in B_{2} .
$$

Então $K_{1}=\overline{\varphi\left(B_{1}\right)}$ e $K_{2}=\overline{\varphi\left(B_{2}\right)}$ são conjuntos compactos, convexos e disjuntos em $\mathbb{C}$.

Agora podemos aplicar o Teorema de Runge, considerando o conjunto compacto dado por $K=K_{1} \cup K_{2} \subset \mathbb{C}$ (não é difícil ver que $\mathbb{C} \backslash K$ é conexo por caminhos), e obter $q \in \mathcal{P}(\mathbb{C})$ tal que $q \sim 1$ em $K_{1}$ e $q \sim 0$ em $K_{2}$.

Portanto o polinômio $h=q \circ \varphi: E \rightarrow \mathbb{C} \in \mathcal{P}(E)$ satisfaz: $h \sim 1$ em $B_{1}$ e $h \sim 0$ em $B_{2}$, no sentido de que $\sup _{x \in B_{1}}|h(x)-1|<\epsilon / 2 M$ e $\sup _{x \in B_{2}}|h(x)|<\epsilon / 2 M$.

Finalmente, $p=p_{1} \cdot h+p_{2} \cdot(1-h)$ é o polinômio que estamos procurando.

De fato, temos:

$$
\sup _{x \in B_{1} \cup B_{2}}|p(x)-f(x)| \leq \max \left\{\sup _{x \in B_{1}}|p(x)-f(x)|, \sup _{x \in B_{2}}|p(x)-f(x)|\right\} .
$$

Se $x \in B_{1}$ então $|p(x)-f(x)|=\left|p_{1}(x) \cdot h(x)+p_{2}(x) \cdot(1-h(x))-f(x)\right| \leq$ $\leq\left(\left|p_{1}(x)\right|+\left|p_{2}(x)\right|\right) \cdot|h(x)-1|+\left|p_{1}(x)-f(x)\right|<M \cdot \epsilon / 2 M+\epsilon / 2=\epsilon$.

Se $x \in B_{2}$ então $|p(x)-f(x)|=\left|p_{1}(x) \cdot h(x)+p_{2}(x) \cdot(1-h(x))-f(x)\right| \leq$ $\leq\left(\left|p_{1}(x)\right|+\left|p_{2}(x)\right|\right) \cdot|h(x)|+\left|p_{2}(x)-f(x)\right|<M \cdot \epsilon / 2 M+\epsilon / 2=\epsilon$.

Assim, nós temos $\sup _{x \in B_{1} \cup B_{2}}|p(x)-f(x)| \leq \epsilon$.

O Corolário seguinte resulta da aplicação sucessiva do Teorema 5.2 ( $n-1$ vezes se temos $n$ bolas). 
Corolário 1. Sejam $B_{1}, B_{2}, \ldots, B_{n}$ bolas fechadas (duas a duas) disjuntas em um espaço de Banach $E$, tais que para cada $j=1,2, \ldots, n-1$ existe uma bola fechada $A_{j}$ com $B_{1} \cup \ldots \cup B_{j} \subset A_{j} \quad e \quad A_{j} \cap B_{j+1}=\phi$. Se $f$ é holomorfa e limitada em uma vizinhança uniforme de $B_{1} \cup B_{2} \cup \ldots \cup B_{n}$, então $f$ pode ser uniformemente aproximada por polinômios em $B_{1} \cup B_{2} \cup \ldots \cup B_{n}$, ou seja, dado $\epsilon>0$, existe $p \in \mathcal{P}(E)$ tal que $\sup _{x \in B_{1} \cup \ldots \cup B_{n}}|p(x)-f(x)|<\epsilon$.

De fato, dado $\epsilon>0$, para cada $j=1,2, \ldots, n-1$, usamos no Teorema a condição $A_{j} \cap B_{j+1}=\phi$ atendida pelas bolas e podemos assim (considerando $\epsilon / n$ em cada etapa da aplicação do Teorema) obter $p_{j} \in \mathcal{P}(E)$ tal que $\sup _{x \in B_{1} \cup \ldots \cup B_{j+1}}\left|p_{j}(x)-f(x)\right|<\frac{j \cdot \epsilon}{n}$. $O$ resultado segue após $n-1$ etapas.

Agora, usando o Teorema 5.2 e seu Corolário, temos condição de estender alguns resultados já conhecidos sobre hiperciclicidade de translações em $H(\mathbb{C})$ para espaços mais gerais de funções inteiras. Porém, antes de fazermos isso, vamos "dar uma olhada" nos espaços de funções para os quais obteremos nossos resultados sobre hiperciclicidade.

Primeiramente, tomamos um espaço de Banach $E$ qualquer e tentamos as translações sobre $H(E)$. Porém, uma rápida olhada no Teorema 5.2 (precisamos que nossas funções sejam limitadas em vizinhanças uniformes de bolas) e argumentos futuros de continuidade uniforme nas bolas do espaço considerado nos levam à álgebra de Fréchet $H_{b}(E)$ (funções inteiras de "tipo limitado" - limitadas nos conjuntos limitados - em $E$, com a topologia da convergência uniforme nos conjuntos limitados, ou seja, nas bolas de $E$ ). É claro que esse ajuste não é necessário se $E$ tem dimensão finita (neste caso temos $H_{b}(E)=H(E)$ ).

Mas surge outro problema quando $E$ tem dimensão infinita, pois separabilidade é uma condição necessária em qualquer discussão sobre hiperciclicidade. Se $E$ tem dimensão infinita, não podemos garantir que $H_{b}(E)$ seja separável (embora haja alguns casos conhecidos para os quais isso ocorre). Desta forma, estamos lidando com subespaços separáveis e fechados (afinal de contas nós precisamos que os espaços sejam completos) de $H_{b}(E)$ e esses subespaços precisam ser invariantes por translações (para que as translações estejam bem definidas como operadores lineares e contínuos sobre esses subespaços).

Portanto, nossos resultados sobre translações serão válidos em subespaços separáveis, fechados e invariantes por translações de $H_{b}(E)$, sendo $E$ espaço de Banach. 
Para resumir, vamos enumerar a seguir alguns exemplos concretos:

1) $E$ com dimensão finita: $H\left(\mathbb{C}^{n}\right)$;

2) Dimensão de $E$ infinita, com $H_{b}(E)$ separável: $H_{b}(E), E=c_{0}, E=$ Tsirelson ;

3 ) Se $E$ é um espaço de Banach tal que $E^{\prime}$ é separável, então $H_{b c}(E)$ (funções em $H_{b}(E)$ de "tipo compacto" - funções de tipo limitado cujos polinômios de Taylor estão no fecho dos polinômios de tipo finito) é um subespaço separável e fechado de $H_{b}(E)$. Mas também precisamos ter subespaços invariantes por translações. Assim, se requerermos que $E^{\prime}$ tenha a propriedade de aproximação, teremos $H_{b c}(E)=H_{w u}(E)$ (funções fracamente-uniformemente contínuas nas bolas de $E$ ) [4], p.56, e não é difícil perceber que $H_{w u}(E)$ é invariante por translações.

Finalmente, uma condição adicional deve ser destacada:

Observação (sobre o Teorema 5.2): Condição (*): Como usaremos o Teorema 5.2 (e seu Corolário) ? Será considerada um determinada função $f$, holomorfa em vizinhanças de bolas fechadas disjuntas. Esta função $f$ será usualmente constituída de partes de outras funções do espaço no qual estaremos trabalhando, cada parte definida na vizinhança correspondente de cada bola considerada. Aplicando o Teorema 5.2, poderemos obter um polinômio (logo, uma função inteira) o qual aproxima $f$ nas bolas.

Contudo, poderemos estar trabalhando com um espaço que pode não conter todos os polinômios (por exemplo, a classe de espaços destacada em 3, nos exemplos anteriores) e como é crucial produzir uma função neste espaço, devemos pedir que o espaço satisfaça uma condição adicional para garantir isso.

Para encontrarmos uma condição razoável, vamos "olhar" a demonstração do Teorema 5.2 mais de perto: O polinômio (o qual queremos que esteja em nosso espaço) produzido é dado por: $p=p_{1} \cdot h+p_{2} \cdot(1-h)$. Cada parcela desta soma deve estar em nosso espaço. Como $h(\mathrm{e}(1-h))$ é um polinômio de tipo finito, $p_{1}$ e $p_{2}$ devem produzir polinômios no espaço considerado quando multiplicados por polinômios de tipo finito. Recordemos que $p_{1}$ e $p_{2}$ surgem na demonstração como aproximações de Taylor para $f$ nas respectivas bolas.

Encontramos portanto nossa Condição (*): "Dizemos que um espaço $X \subset H(E)$, $E$ Banach, satisfaz a Condição (*) se os polinômios de Taylor das funções em $X$ podem ser aproximados por polinômios cujos produtos por polinômios de tipo finito ainda estão 
no espaço $X . "$

Embora possa parecer um pouco confusa, a Condição (*) se mostra bem razoável, à luz de nossos exemplos previamente conhecidos: Os casos 1) $H\left(\mathbb{C}^{n}\right)$ e 2) $H_{b}(E)$ separável são triviais, uma vez que ambos os tipos de espaço contêm todos os polinômios. O terceiro grupo de exemplos também atende à Condição $\left(^{*}\right)$, por sua própria definição: Polinômios de Taylor de funções em $H_{b c}(E)$ estão no fecho (portanto podem ser aproximados por) dos polinômios de tipo finito!

O primeiro resultado que iremos estender é o de Birkhoff, sobre a hiperciclicidade de operadores de translação. Para tal, iremos simplesmente adaptar a prova de Aron e Markose [5] ao nosso Teorema 5.2.

Teorema 5.3. Sejam $E$ um espaço de Banach e $X$ um subespaço fechado, separável $e$ invariante por translações de $H_{b}(E)$. Suponhamos também que o espaço $X$ satisfaça à Condição $\left(^{*}\right)$. Se $v \neq 0$ é qualquer vetor não-nulo em $E$, então o operador de translação por $v, \tau_{v}: X \rightarrow X$, dado por $\tau_{v}(f)(x)=f(x+v)$, é hipercíclico.

Demonstração:

Seja $\left(g_{j}\right)_{j}$ uma sequência densa em $X$ tal que cada $g_{j}$ ocorre uma quantidade infinita de vezes na sequência. Dado $v \neq 0$ em $E$, queremos mostrar que a translação por $v, \tau_{v}: X \rightarrow X$, é hipercíclica.

Seja $\left(D_{j}\right)_{j}$ uma sequência de bolas fechadas e disjuntas em $E$, cada $D_{j}$ com raio $j$ e centro $c_{j}=n_{j} . v$, com $n_{j} \in \mathbb{N}, n_{1}<n_{2}<n_{3}<\ldots$

Não é difícil ver que existe uma sequência $\left(E_{j}\right)_{j}$ de bolas fechadas (em $E$ ) centradas na origem e tais que $D_{1} \cup D_{2} \cup \ldots \cup D_{j} \subset E_{j}$ e $E_{j} \cap D_{j+1}=\phi$ para todo $j \in \mathbb{N}$.

Fixemos qualquer (polinômio) $Q_{1} \in X$.

Como $E_{1} \cap D_{2}=\phi \quad$ (bolas fechadas disjuntas), segue do Teorema 5.2 que existe um polinômio $Q_{2} \in X$ (para isso a Condição $\left(^{*}\right)$ está sendo considerada) tal que

$$
\sup _{x \in E_{1}}\left|Q_{2}(x)\right|<\frac{1}{2} \quad \text { e } \sup _{x \in D_{2}}\left|Q_{2}(x)-\left[g_{1}\left(x-c_{2}\right)-Q_{1}(x)\right]\right|<\frac{1}{2} \text {. }
$$

De fato, consideremos (no Teorema 5.2)

$$
f(x)=\left\{\begin{array}{cl}
0 & \text { em uma vizinhança uniforme de } E_{1} \\
g_{1}\left(x-c_{2}\right)-Q_{1}(x) & \text { em uma vizinhança uniforme de } D_{2} .
\end{array}\right.
$$


A seguir, usando novamente o Teorema 5.2, tomemos um polinômio $Q_{3} \in X$ tal que

$$
\sup _{x \in E_{2}}\left|Q_{3}(x)\right|<\frac{1}{2^{2}} \quad \text { e } \sup _{x \in D_{3}}\left|Q_{3}(x)-\left[g_{2}\left(x-c_{3}\right)-Q_{1}(x)-Q_{2}(x)\right]\right|<\frac{1}{2^{2}} .
$$

Prosseguindo desta forma, construimos uma sequência de polinômios $\left(Q_{n}\right)_{n} \subset X$ tal que para cada $n \geq 2$ :

$$
\begin{gathered}
\text { (I) } \sup _{x \in E_{n-1}}\left|Q_{n}(x)\right|<\frac{1}{2^{n-1}} \\
\text { (II) } \sup _{x \in D_{n}}\left|Q_{n}(x)-\left[g_{n-1}\left(x-c_{n}\right)-\sum_{j=1}^{n-1} Q_{j}(x)\right]\right|<\frac{1}{2^{n-1}} \text {. }
\end{gathered}
$$

De (I), temos que a sequência $\left(\sum_{j=1}^{n} Q_{j}\right)_{n}$ é uma sequência de Cauchy em $X$.

Podemos considerar então $f=\sum_{j=1}^{\infty} Q_{j} \in X$ ( $f$ é nossa candidata para ser hipercíclica com relação a $\tau_{v}$ ).

Dados $R>0, \epsilon>0$ e $g=g_{j_{0}} \in\left(g_{j}\right)_{j}$, iremos mostrar que existe $l \in \mathbb{N}$ tal que

$$
\sup _{\|x\| \leq R}\left|f\left(x+c_{l}\right)-g(x)\right|<\epsilon
$$

Como $\left(g_{j}\right)_{j}$ é denso em $X$ e $c_{j}=n_{j} v$, isto significa que $\left\{\tau_{n_{j} v} f\right\}_{j}$ é denso em $X$ e portanto $f$ é hipercíclica para $\tau_{v}$.

De fato, podemos tomar $l \in \mathbb{N}$ (suficientemente grande) tal que $l>R, \frac{1}{2^{l-1}}<\frac{\epsilon}{2}$ e $g=g_{l-1}$.

Se $\|x\| \leq R$, então $w=x+c_{l} \in B[0 ; R]+c_{l} \subset B[0 ; l]+c_{l}=D_{l} \subset E_{l}$.

Logo

$$
\begin{gathered}
\sup _{\|x\| \leq R}\left|f\left(x+c_{l}\right)-g(x)\right| \leq \sup _{w \in D_{l}}\left|f(w)-g_{l-1}\left(w-c_{l}\right)\right| \leq \\
\leq \sup _{w \in D_{l}}\left|f(w)-\sum_{j=1}^{l} Q_{j}(w)\right|+\sup _{w \in D_{l}}\left|\left[\sum_{j=1}^{l} Q_{j}(w)\right]-g_{l-1}\left(w-c_{l}\right)\right| .
\end{gathered}
$$


Na primeira parcela da expressão à direita, temos

$$
\begin{aligned}
& \sup _{w \in D_{l}}\left|f(w)-\sum_{j=1}^{l} Q_{j}(w)\right| \leq \sup _{w \in E_{l}}\left|\sum_{j=l+1}^{\infty} Q_{j}(w)\right| \leq \\
& \leq \sum_{j=l+1}^{\infty} \sup _{w \in E_{j-1}}\left|Q_{j}(w)\right|<\frac{1}{2^{l}}+\frac{1}{2^{l+1}}+\ldots=\frac{1}{2^{l-1}}<\frac{\epsilon}{2} .
\end{aligned}
$$

Para a segunda parcela, de (II) temos:

$$
\sup _{w \in D_{l}}\left|\left[\sum_{j=1}^{l} Q_{j}(w)\right]-g_{l-1}\left(w-c_{l}\right)\right|<\frac{1}{2^{l-1}}<\frac{\epsilon}{2} .
$$

Portanto, $\sup _{\|x\| \leq R}\left|f\left(x+c_{k}\right)-g(x)\right|<\epsilon$, como requerido.

O segundo resultado de extensão que queremos mostrar refere-se à existência de funções hipercíclicas comuns a coleções não-enumeráveis de translações.

É sabido que todo operador hipercíclico sobre um espaço de Fréchet tem um conjunto residual (contém um conjunto $G_{\delta}$ denso no espaço) de vetores hipercíclicos (Teorema 1.13). Como interseções enumeráveis de conjuntos residuais são ainda residuais, é fácil ver que famílias enumeráveis de operadores hipercíclicos admitem vetores hipercíclicos comuns (vetores os quais são hipercíclicos para todos os operadores da família).

$\mathrm{O}$ assunto se torna mais interessante quando começamos a pensar sobre famílias não enumeráveis de operadores hipercíclicos e a questionar se tais famílias admitem ou não vetores hipercíclicos comuns. Em [18], Costakis e Sambarino provaram que existe um conjunto residual de funções hipercíclicas comuns a todas as translações (não-nulas) em $H(\mathbb{C})$. Em sua prova, eles usaram fortemente o Teorema de Runge (Teorema 5.1), tomando como compactos certas coleções finitas de bolas fechadas disjuntas, e sua técnica é relacionada com uma certa decomposição unidimensional de $\mathbb{C}$.

Desta forma, é natural perguntar sobre como podemos utilizar o Corolário do Teorema 5.2 para estender este resultado de Costakis e Sambarino.

O resultado que obtivemos é o seguinte: 
Teorema 5.4. Sejam E um espaço de Banach e $X$ um subespaço fechado, separável $e$ invariante por translações de $H_{b}(E)$. Suponhamos também que o espaço $X$ satisfaça $\grave{a}$ Condição (*). Para cada $v \neq 0$ em E, a familia (não-enumerável) de translações $\left\{\tau_{c \cdot v} ; c \neq 0\right.$ em $\left.\mathbb{C}\right\}$ admite um conjunto residual de funções hipercíclicas comuns.

Assim como o resultado análogo de Costakis e Sambarino em [18], o Teorema 5.4 é uma consequência imediata de dois teoremas, os quais descreveremos a seguir. Devemos mencionar que as demonstrações são essencialmente as de Costakis e Sambarino para a existência de um conjunto residual de funções hipercíclicas comuns para todas as translações não-nulas sobre $H(\mathbb{C})$, mas agora nosso Corolário do Teorema 5.2 se encaixa perfeitamente nessas demonstrações, substituindo o Teorema de Runge (Teorema 5.1) e nos permitindo obter um resultado "similar" (num certo sentido) para classes mais gerais de espaços.

Teorema 5.5. Sejam E um espaço de Banach e $X$ um subespaço fechado, separável e invariante por translações de $H_{b}(E)$. Suponhamos também que o espaço $X$ satisfaça à Condição $\left(^{*}\right)$. Se v é um vetor unitário em E, então o conjunto das funções hipercíclicas comuns a todas as translações $\tau_{e^{2 \pi i \theta} \cdot v}: X \rightarrow X, \theta \in[0,1]$, é residual (contém um conjunto $G_{\delta}$ denso no espaço) em $X$.

Demonstração:

Seja $\left(g_{j}\right)_{j}$ uma sequência densa em $X$.

Para cada $s, j, k, m \in \mathbb{N}$, definamos o conjunto

$$
\begin{array}{r}
E(s, j, k, m)=\{f \in X ; \text { para todo } \theta \in[0,1], \text { existe } n=n(\theta) \leq m \\
\left.\quad \text { tal que } \sup _{\|x\| \leq k}\left|f\left(x+n e^{2 \pi i \theta} \cdot v\right)-g_{j}(x)\right|<\frac{1}{s}\right\} .
\end{array}
$$

Afirmação: Cada $E(s, j, k, m)$ é aberto em $X$.

Para provar isto, fixemos $s, j, k, m \in \mathbb{N}$ e consideremos $f \in E(s, j, k, m)$. 
Para $l=1, \ldots, m$, defina:

$$
C_{l}=\left\{\alpha \in S^{1} ; \sup _{\|x\| \leq k}\left|f(x+l \alpha v)-g_{j}(x)\right|<\frac{1}{s}\right\} .
$$

A função $\varphi_{l}: S^{1} \rightarrow \mathbb{R}$ dada por $\varphi_{l}(\alpha)=\sup _{\|x\| \leq k}\left|f(x+l \alpha v)-g_{j}(x)\right|$ é contínua, logo cada $C_{l}$ é aberto em $S^{1}$.

Como $f \in E(s, j, k, m)$, temos que $S^{1}=\bigcup_{l=1}^{m} C_{l}$ é uma cobertura aberta do
junto compacto $S^{1} \subset \mathbb{C}$.

Existem então conjuntos compactos $I_{l} \subset C_{l}, l=1, \ldots, m$, tais que $S^{1}=\bigcup_{l=1}^{m} I_{l}$.

Cada $I_{l} \subset C_{l}$ é compacto e daí segue que $\varphi_{l}(\alpha) \leq a_{l}<\frac{1}{s}$ para todo $\alpha \in I_{l}$.

Considere agora $\epsilon_{l}=\frac{1}{s}-a_{l}, l=1, \ldots, m$, e tome $\epsilon=\min \left\{\epsilon_{1}, \ldots, \epsilon_{m}\right\}>0$.

$$
V=\left\{g \in X ; \sup _{\|x\| \leq k+m}|g(x)-f(x)|<\epsilon\right\} \text { é uma vizinhança aberta de } f \text { em } X \text {. }
$$

Seja $g \in V$. Dado $\alpha \in S^{1}$, temos $\alpha \in I_{l_{0}}$ para algum $l_{0} \in\{1, \ldots, m\}$ e $\|x\| \leq k$ implica $\left\|x+l_{0} \alpha v\right\| \leq\|x\|+l_{0} \leq k+m$.

Temos então:

$$
\begin{gathered}
\sup _{\|x\| \leq k}\left|g\left(x+l_{0} \alpha v\right)-g_{j}(x)\right| \leq \sup _{\|x\| \leq k}\left|g\left(x+l_{0} \alpha v\right)-f\left(x+l_{0} \alpha v\right)\right|+ \\
+\sup _{\|x\| \leq k}\left|f\left(x+l_{0} \alpha v\right)-g_{j}(x)\right| \leq \sup _{\|w\| \leq k+m}|g(w)-f(w)|+a_{l_{0}}<\epsilon+a_{l_{0}} \leq \frac{1}{s} .
\end{gathered}
$$

Portanto $g \in E(s, j, k, m)$, o que implica $V \subset E(s, j, k, m)$ e podemos concluir que $E(s, j, k, m)$ é aberto.

Afirmação: $\bigcup_{m \in \mathbb{N}} E(s, j, k, m)$ é denso em $X$, para cada $s, j, k \in \mathbb{N}$. 
De fato, fixemos $s, j, k \in \mathbb{N}$ e seja $h \in X$.

Dados $R>0$ e $\epsilon>0$, queremos encontrar $f \in X$ e $m \in \mathbb{N}$ tais que $f \in E(s, j, k, m)$ e $\sup _{\|x\| \leq R}|f(x)-h(x)|<\epsilon$.

Como $E\left(s, j, k_{1}, m\right) \subset E\left(s, j, k_{2}, m\right)$ se $k_{1}>k_{2}$, podemos assumir $k>R$.

Primeiramente escolha $\delta<\frac{1}{2}$ tal que

$$
\|x\| \leq k,\|x-w\|<\delta \Rightarrow\left|g_{j}(x)-g_{j}(w)\right|<\frac{1}{2 s}
$$

(isto é possível porque nossa $g_{j}$, estando em $H_{b}(E)$, é uniformemente contínua nos conjuntos limitados).

Seja $B=\{x \in E ;\|x\| \leq k+\delta\}$ e fixemos $t=2 k+1$.

Para qualquer partição (a ser escolhida mais tarde) $0=\theta_{0}<\theta_{1}<\ldots<\theta_{l}=1$ de $[0,1]$, defina:

$$
B_{d}=B+(d+1) t e^{2 \pi i \theta_{d}} \cdot v \quad(d=0,1, \ldots, l) .
$$

Note que cada $B_{d}$ é uma translação da bola fechada $B$ (logo cada bola tem raio $k+\delta)$ e $B, B_{0}, B_{1}, \ldots, B_{l}$ são bolas fechadas duas a duas disjuntas, já que as distâncias entre os centros são maiores do que $2(k+\delta)$.

Consideremos agora a função $q$ dada por:

$$
q(x)=\left\{\begin{array}{lc}
h(x) & \text { em uma vizinhança uniforme de } B \\
g_{j}\left(x-(d+1) t e^{2 \pi i \theta_{d}} \cdot v\right) & \text { em uma vizinhança uniforme de } B_{d} . \\
& (d=0,1, \ldots, l)
\end{array}\right.
$$

Pelo Corolário do Teorema 5.2, podemos produzir um polinômio $f \in X$ (para isso a Condição $\left(^{*}\right)$ está sendo usada, bem como o fato de que $h$ e $g_{j}$ - e suas translações - estão em $X$ ) tal que:

$$
\sup _{x \in S}|f(x)-q(x)|<\min \left\{\frac{1}{2 s}, \epsilon\right\} \quad\left(S=B \cup B_{0} \cup B_{1} \cup \ldots \cup B_{l}\right) .
$$


Portanto, temos:

$$
\sup _{\|x\| \leq R}|f(x)-h(x)| \leq \sup _{\|x\| \leq k}|f(x)-h(x)| \leq \sup _{x \in B}|f(x)-q(x)|<\epsilon,
$$

uma vez que $B \subset S$.

Tudo o que precisamos fazer agora é escolher uma partição $0=\theta_{0}<\theta_{1}<\ldots<\theta_{l}=1$ de $[0,1]$ tal que nossa $f$ esteja em $\cup_{m} E(s, j, k, m)$.

Para tal, vamos mostrar ser possível obter uma partição tal que, dado $\theta \in[0,1]$, $\theta_{d} \leq \theta \leq \theta_{d+1}$, tenhamos

$$
\sup _{\|x\| \leq k}\left|f\left(x+(d+1) t e^{2 \pi i \theta} \cdot v\right)-g_{j}(x)\right|<\frac{1}{s} .
$$

Desta forma, nossos $m$ e $n=n(\theta)$ na definição de $E(s, j, k, m)$ serão dados por $n(\theta)=(d+1) t \leq(l+1) t=m$.

Assim, se pudermos tomar uma partição que assegure (I), fazemos $m=(l+1) t \in \mathbb{N}$ e então $f \in E(s, j, k, m)$.

Afirmamos que $0=\theta_{0}<\theta_{1}<\ldots<\theta_{l}=1$ servirão se essa partição satisfizer a seguinte condição:

$$
(d+1) t \cdot\left|e^{2 \pi i \theta_{d+1}}-e^{2 \pi i \theta_{d}}\right|<\delta \quad(d=0, \ldots, l-1) .
$$

De fato, suponhamos que (II) é satisfeita. Se $\|x\| \leq k$, então

$$
\left\|x+(d+1) t e^{2 \pi i \theta} \cdot v-(d+1) t e^{2 \pi i \theta_{d}} \cdot v\right\| \leq k+(d+1) t \cdot\left|e^{2 \pi i \theta}-e^{2 \pi i \theta_{d}}\right| .
$$

Como $\left|e^{2 \pi i \theta}-e^{2 \pi i \theta_{d}}\right| \leq\left|e^{2 \pi i \theta_{d+1}}-e^{2 \pi i \theta_{d}}\right|$ porque $\theta_{d} \leq \theta<\theta_{d+1}$ (e podemos supor $\left|\theta_{d+1}-\theta_{d}\right|<1 / 2$ para todo $d$ ), então temos, de (II)

$$
\|x\| \leq k \Rightarrow x+(d+1) t e^{2 \pi i \theta} \cdot v \in B_{d} .
$$

Assim, usando $\alpha, \beta, \gamma$, temos

$$
\sup _{\|x\| \leq k}\left|f\left(x+(d+1) t e^{2 \pi i \theta} \cdot v\right)-g_{j}(x)\right| \leq
$$




$$
\begin{aligned}
& \leq \sup _{\|x\| \leq k}\left|f\left(x+(d+1) t e^{2 \pi i \theta} \cdot v\right)-g_{j}\left(x+(d+1) t e^{2 \pi i \theta} \cdot v-(d+1) t e^{2 \pi i \theta_{d}} \cdot v\right)\right|+ \\
& +\sup _{\|x\| \leq k}\left|g_{j}\left(x+(d+1) t e^{2 \pi i \theta} \cdot v-(d+1) t e^{2 \pi i \theta_{d}} \cdot v\right)-g_{j}(x)\right|<\frac{1}{2 s}+\frac{1}{2 s}=\frac{1}{s},
\end{aligned}
$$

o que prova (I).

Precisamos portanto estabelecer (II).

Para isto, é claro que $0=\theta_{0}<\theta_{1}<\ldots<\theta_{l}=1 \mathrm{com}$

$$
\left(\theta_{d+1}-\theta_{d}\right)<\frac{\delta}{2 \pi t(d+1)} \quad(d=0,1, \ldots, l-1)
$$

é suficiente, pois $\left|e^{2 \pi i \theta_{d+1}}-e^{2 \pi i \theta_{d}}\right| \leq 2 \pi\left(\theta_{d+1}-\theta_{d}\right)$.

Portanto, ao mesmo tempo em que as distâncias entre $\theta_{d}$ e $\theta_{d+1}$ devem ser suficientemente pequenas para satisfazer estas relações, devemos ter $\sum_{d=0}^{l-1}\left(\theta_{d+1}-\theta_{d}\right)=1$ (afinal de contas precisamos cobrir o intervalo para conseguirmos a partição).

E isto será possível porque $\sum_{n=1}^{\infty} \frac{1}{n}=+\infty$.

De fato, escolha $l \in \mathbb{N}$ tal que $\eta=\frac{\delta}{2 \pi t} \cdot\left(1+\frac{1}{2}+\ldots+\frac{1}{l}\right)>1$ e tome

$$
\theta_{d+1}-\theta_{d}=\frac{1}{\eta}\left(\frac{\delta}{2 \pi t(d+1)}\right) \quad(d=0,1, \ldots, l-1) .
$$

Esta escolha dos $\theta$ s é suficiente.

Podemos agora finalmente concluir a prova do Teorema.

Como cada $E(s, j, k, m)$ é aberto em $X$, cada $\bigcup_{m \in \mathbb{N}} E(s, j, k, m)$ é denso em $X$ e $X$ é Fréchet (logo Baire), o conjunto

$$
G=\bigcap_{s \in \mathbb{N}} \bigcap_{j \in \mathbb{N}} \bigcap_{k \in \mathbb{N}} \bigcup_{m \in \mathbb{N}} E(s, j, k, m)
$$

é um subconjunto $G_{\delta}$ denso (logo não-vazio) em $X$. 
Seja $f \in G$.

Dados $\theta \in[0,1], R>0, \epsilon>0$, e $h \in X$, tome números naturais $k>R, s>\frac{2}{\epsilon}$ e $j$ tais que $\sup _{\|x\| \leq R}\left|g_{j}(x)-h(x)\right|<\frac{\epsilon}{2}$.

Como $f \in G$, existe $m \in \mathbb{N}$ tal que $f \in E(s, j, k, m)$.

Então existe $n=n(\theta) \leq m$ com $\sup _{\|x\| \leq k}\left|f\left(x+n e^{2 \pi i \theta} \cdot v\right)-g_{j}(x)\right|<\frac{1}{s}<\frac{\epsilon}{2}$.

e daí segue que $\sup _{\|x\| \leq R}\left|f\left(x+n e^{2 \pi i \theta} \cdot v\right)-h(x)\right|<\epsilon$.

Portanto $f$ é hipercíclica para $\tau_{e^{2 \pi i \theta} \cdot v}$ para cada $\theta \in[0,1]$ e podemos concluir que o conjunto residual $G$ é formado por vetores hipercíclicos comuns para a família $\left\{\tau_{e^{2 \pi i \theta \cdot v}}\right\}_{\theta \in[0,1]}$.

Os resultados a seguir serão utilizados na demonstração do próximo teorema:

Teorema 5.6. (Ansari, [2]) Sejam $T$ um operador hipercíclico e $n \in \mathbb{N}$. Então $T^{n}$ é também hipercíclico e, mais ainda, $T$ e $T^{n}$ têm os mesmos vetores hipercíclicos.

Se $s \in \mathbb{R}$, vamos denotar por $[s]$ a parte inteira de $s$ e por $\{s\}$ a parte fracionária de $s(s=[s]+\{s\})$.

Lema 5.7. Se $t>0$ é irracional e $0<\gamma<1$, então existe uma sequência $n_{1}<n_{2}<$ $n_{3}<\ldots$ em $\mathbb{N}$ tal que

$$
0<\left\{n_{j} t\right\}<\gamma \quad(\forall j) \quad \text { e } \quad \sup \left(n_{j+1}-n_{j}\right)<+\infty .
$$

Demonstração:

Como $t$ é irracional, sabemos que $\left\{e^{2 \pi i n \cdot t} ; n \in \mathbb{N}\right\}$ é denso em $S^{1}$. Logo o conjunto $\{\{n \cdot t\} ; n \in \mathbb{N}\}$ é denso em $[0,1]$. 
Pela densidade, existe $n_{1}=\min \{n \in \mathbb{N} ; \gamma / 2<\{n t\}<\gamma\}$.

Tome $p_{1}=\min \left\{n \in \mathbb{N} ;\{n t\}<\gamma-\left\{n_{1} t\right\}\right\}$ e defina $n_{2}=n_{1}+p_{1}$.

É claro que $\left\{n_{1} t\right\}<\left\{n_{2} t\right\}<\gamma$ e $n_{1}<n_{2}$.

Defina $p_{2}=\min \left\{n \in \mathbb{N} ; 1-\left\{n_{1} t\right\}<\{n t\}<1-\gamma / 2\right\}$.

Se colocamos $n_{3}=n_{2}+p_{2}$, não é difícil ver que

$$
\left\{n_{3} t\right\}<\gamma / 2<\left\{n_{1} t\right\}<\gamma
$$

Agora passamos de $n_{3}$ para $n_{4}$ do mesmo modo como fizemos de $n_{1}$ para $n_{2}$, ou seja, tomamos $p_{3}=\min \left\{n \in \mathbb{N} ;\{n t\}<\gamma-\left\{n_{3} t\right\}\right\} \leq p_{1} \quad\left(\right.$ pois $\left\{n_{3} t\right\}<\left\{n_{1} t\right\}$ ) e fazemos $n_{4}=n_{3}+p_{3}$. É claro que $\left\{n_{3} t\right\}<\left\{n_{4} t\right\}<\gamma$ e $n_{3}<n_{4}$.

Usamos até agora $p_{1}, p_{2}$ e $p_{3}$ para os acréscimos e temos $p_{3} \leq p_{1}$.

Iremos construir a sequência desejada $\left(n_{j}\right)$ tal que $\sup \left(n_{j+1}-n_{j}\right)=\max \left\{p_{1}, p_{2}\right\}$.

De fato, comparando $\left\{n_{4} t\right\}$ com $\left\{n_{1} t\right\}$, temos duas possibilidades:

(i) $\left\{n_{4} t\right\}<\left\{n_{1} t\right\}$ : Neste caso passamos de $n_{4}$ para $n_{5}$ como fizemos de $n_{1}$ para $n_{2}$. Então teremos $n_{5}=n_{4}+p_{4}$, com $p_{4} \leq p_{1}$ e $\left\{n_{4} t\right\}<\left\{n_{5} t\right\}<\gamma$.

(ii) $\left\{n_{4} t\right\}>\left\{n_{1} t\right\}$ : Neste caso definimos $n_{5}=n_{4}+p_{2}$ (observe que $p_{2}$ não depende de $n_{2}$ ) e teremos $\left\{n_{5} t\right\}<\gamma / 2<\left\{n_{1} t\right\}<\gamma$.

Prosseguindo deste modo, fica claro que podemos construir uma sequência $n_{1}<n_{2}<$ $n_{3}<\ldots$ em $\mathbb{N}$ tal que $0<\left\{n_{j} t\right\}<\gamma$ (para todo $j$ ) e $\sup \left(n_{j+1}-n_{j}\right)<+\infty$ e a prova do Lema está completa.

Através do próximo teorema, combinado com o Teorema 5.5, teremos finalmente a prova do Teorema 5.4:

Teorema 5.8. Sejam $E$ um espaço de Banach e $X$ um subespaço fechado, separável $e$ invariante por translações de $H_{b}(E)$. Suponhamos também que o espaço $X$ satisfaça à 
Condição $\left(^{*}\right)$. Se $v$ é um vetor unitário em $E$ e $f \in X$ é hipercíclica para a translação $\tau_{v}: X \rightarrow X$, então $f$ é hipercíclica para toda translação da família $\left\{\tau_{r \cdot v}\right\}_{r>0}$.

Demonstração:

Seja $f \in X$ hipercíclica para a translação $\tau_{v}: X \rightarrow X$ e seja $r>0$.

Queremos provar que a órbita de $f$ sob $\tau_{r \cdot v}$ é densa em $X$, isto é, dados $g \in X$, $R>0$ e $\epsilon>0$, devemos mostrar que existe $n \in \mathbb{N}$ tal que

$$
\sup _{\|x\| \leq R}|f(x+n r v)-g(x)|<\epsilon .
$$

Iremos separar a demonstração em duas partes:

PARTE I) $r=p / q(p, q \in \mathbb{N})$ é racional:

Como $f$ é hipercíclica para $\tau_{v}$, segue do Teorema de Ansari (Teorema 5.6) que $f$ é também hipercíclica para $\tau_{v}^{p}$, o que implica na existência de $m \in \mathbb{N}$ tal que $\sup _{\|x\| \leq R}|f(x+m p v)-g(x)|<\epsilon$.

Mas $m p=m q r$. Assim, encontramos $m q \in \mathbb{N}$ tal que

$$
\sup _{\|x\| \leq R}|f(x+m q r v)-g(x)|<\epsilon .
$$

E podemos concluir portanto que $f$ é hipercíclica para $\tau_{r v}$.

PARTE II) $r>0$ é irracional:

Seja $\delta>0$ tal que $\|x\| \leq R,\|x-w\|<\delta \Rightarrow|g(x)-g(w)|<\frac{\epsilon}{4}$.

Tome $B=B[0 ; R+\delta]$ e escolha $k \in \mathbb{N}$ tal que $k>2(R+\delta)$.

Como $r / k$ é irracional, podemos concluir (pelo Lema 5.7 anterior) que existe uma sequência $n_{1}<n_{2}<n_{3}<\ldots$ em $\mathbb{N}$ tal que

$$
0<\left\{n_{j} \frac{r}{k}\right\}<\frac{\delta}{k} \quad(\forall j) \quad \text { e } \quad \sup \left(n_{j+1}-n_{j}\right)<+\infty
$$


(considere apenas $t=r / k$ e $\gamma=\delta / k)$.

Se colocarmos $m_{j}=\left[n_{j} \frac{r}{k}\right]$, então

$$
0<n_{j} r-m_{j} k<\delta \quad \text { e } \quad\left(m_{j+1}-m_{j}\right) \leq m \in \mathbb{N} \quad \text { para todo } j .
$$

Para cada $l=1,2, \ldots, m-1$, considere a seguinte translação de $B$ :

$$
B_{l}=B+l k v \text {. }
$$

Uma vez que $k>2(R+\delta)$, é fácil ver que as bolas $B, B_{1}, B_{2}, \ldots, B_{m-1}$ são duas a duas disjuntas.

Consideremos agora o conjunto $S=B \cup B_{1} \cup \ldots \cup B_{m-1}$ e uma função $h$ dada por:

$$
h(x)= \begin{cases}g(x) & \text { em uma vizinhança uniforme de } B \\ g(x-l k v) & \text { em uma vizinhança uniforme de } B_{l} . \\ & (l=1, \ldots, m-1)\end{cases}
$$

Pelo Corolário do Teorema 5.2, existe uma função $\xi \in X$ (para isto a Condição (*) está sendo usada) tal que:

$$
\sup _{x \in S}|\xi(x)-h(x)|<\frac{\epsilon}{4} \text {. }
$$

Pelo Teorema de Ansari (5.6), temos $f$ hipercíclica para $\tau_{v}$ implica $f$ hipercíclica para $\tau_{v}^{k}$.

Existe então $n \in \mathbb{N}$ tal que

$$
\sup _{x \in S}|f(x+n k v)-\xi(x)|<\frac{\epsilon}{4} .
$$

Como $m_{j+1}-m_{j} \leq m$ para todo $j$, então existe $j \in \mathbb{N}$ tal que $n \leq m_{j} \leq n+(m-1)$.

Então $m_{j} k=n k+l k$ para algum $l \in\{0,1, \ldots, m-1\}$ e se colocamos $w=\left(n_{j} r-m_{j} k\right) v$, temos $\|w\|=n_{j} r-m_{j} k<\delta$.

Finalmente, se $\|x\| \leq R$, então temos:

$$
\left|f\left(x+n_{j} r v\right)-g(x)\right|=\left|f\left(x+m_{j} k v+\left(n_{j} r-m_{j} k\right) v\right)-g(x)\right| \leq
$$




$$
\begin{gathered}
\leq|f(x+(n k+l k) v+w)-\xi(x+w+l k v)|+ \\
+|\xi(x+w+l k v)-g(x+w)|+ \\
+|g(x+w)-g(x)| .
\end{gathered}
$$

Agora, temos:

$x+w+l k v \in B_{l} \stackrel{(\mathbf{3})}{\Rightarrow}|f(x+(n k+l k) v+w)-\xi(x+w+l k v)|<\frac{\epsilon}{4}$

$x+w+l k v \in B_{l} \stackrel{(2)}{\Rightarrow}|\xi(x+w+l k v)-g(x+w)|<\frac{\epsilon}{4} ;$

$\|w\|<\delta,\|x\| \leq R \stackrel{(\mathbf{1})}{\Rightarrow}|g(x+w)-g(x)|<\frac{\epsilon}{4}$.

Portanto, encontramos $n_{j} \in \mathbb{N}$ tal que

$$
\sup _{\|x\| \leq R}\left|f\left(x+n_{j} r v\right)-g(x)\right|<\epsilon
$$

e podemos concluir que $f$ é hipercíclica para $\tau_{r v}$.

Finalmente, observe que o Teorema 5.4 é obtido combinando-se o Teorema 5.5 e o Teorema 5.8. 


\section{Algumas notações e definições}

$[s](s \in \mathbb{R})=$ parte inteira de $s$ (maior inteiro que é menor ou igual a $s$ ).

$\{s\} \quad(s \in \mathbb{R})=$ parte fracionária de $s(\{s\}=s-[s])$.

$B[x ; R]=$ bola fechada de centro $x$ e raio $R>0$ (em um espaço métrico).

$B(x ; R)=$ bola aberta de centro $x$ e raio $R>0$ (em um espaço métrico).

$B_{n}[f ; R]=\left\{g \in H(\mathbb{C}) ; p_{n}(g-f) \leq R\right\}$.

$B_{n}(f ; R)=\left\{g \in H(\mathbb{C}) ; p_{n}(g-f)<R\right\}$.

BAIRE (ESPAÇO DE) - um espaço topológico $X$ é um espaço de Baire quando todo conjunto aberto e não-vazio em $X$ é de $2^{a}$ categoria.

$1^{a}$ CATEGORIA (CONJUNTO DE) - $B \subset X$ (espaço topológico) é de $1^{a}$ categoria se, se somente se, $B$ é união enumerável de conjuntos "raros".

$2^{a}$ CATEgoria (CONJUNTO DE) - $B \subset X$ (espaço topológico) é de $2^{a}$ categoria quando não é de $1^{a}$ categoria.

C.H. = CRITÉRIO DE HIPERCICLICIDADE.

CÍCLICO (VETOR) - Definição 1.10.

COMPACTO-ABERTA (TOPOLOGIA) - em $H\left(\mathbb{C}^{n}\right)$, é a topologia gerada pelas seminormas $p_{K}(f)=\sup _{z \in K}|f(z)|$, sendo $K$ (compacto) $\subset \mathbb{C}^{n}$, ou seja, é a topologia da covergência uniforme sobre os conjuntos compactos de $\mathbb{C}^{n}$.

CONDIÇÃO $\left({ }^{*}\right)$ - um espaço $X \subset H(E), E$ Banach, satisfaz a Condição $(*)$ se os polinômios de Taylor das funções em $X$ podem ser aproximados por polinômios cujos 
produtos por polinômios de tipo finito ainda estão no espaço $X$.

CONSTANTE DE POROSIDADE - Definição 4.1.

CONSTANTE DE $t$-POROSIDADE - Definição 4.3.

CONVOLUÇÃO (OPERADOR DE) - Teorema 2.3.

CRITÉRIO DE HIPERCICLICIDADE - Teorema 1.14.

DENSAMENTE UNIVERSAL - Definição 1.5.

ESPAÇABILIDADE - propriedade relativa a um conjunto que contenha um espaço vetorial fechado de dimensão infinita, a menos (possivelmente) do vetor nulo.

ESPAÇÁVEL - Definição 2.1.

$G_{\delta}=$ interseção enumerável de conjuntos abertos.

GENERICAMENTE UNIVERSAL - Definição 1.5.

$H(E)=$ espaço das funções inteiras em $E$ (Banach), ou seja, funções $f: E \rightarrow \mathbb{C}$ que são holomorfas (analíticas) em todo $E$.

$H_{b}(E)=$ álgebra de Fréchet das funções inteiras e de "tipo limitado" - limitadas em conjuntos limitados - em $E$, com a topologia da convergência uniforme nos conjuntos limitados (ou equivalentemente nas bolas) de $E$.

$H_{b c}(E)=$ funções em $H_{b}(E)$ de "tipo compacto" - funções de tipo limitado cujos polinômios de Taylor estão no fecho dos polinômios de "tipo finito". $H_{b c}(E)$ é subespaço fechado de $H_{b}(E)$.

$H_{w u}(E)=$ subespaço de $H(E)$ formado pelas das funções (inteiras) que são fracamente uniformemente contínuas em cada conjunto limitado de $E$.

$\mathcal{H C}(T)=$ conjunto dos vetores hipercíclicos para o operador $T$.

HIPERCÍCLICO (OPERADOR, VETOR) - Definição 1.9.

LINEABILIDADE - propriedade relativa a um conjunto que contenha um espaço vetorial de dimensão infinita, a menos (possivelmente) do vetor nulo.

LINEÁVEL - Definição 2.1.

$\mathcal{P}(E)=$ espaço dos polinômios sobre $E$ (Banach). 
$p_{n}(f)=\sup _{|z| \leq n}|f(z)|, f \in H(\mathbb{C}), n \in \mathbb{N}$.

POROSO (CONJUNTO) - Definição 4.1.

$\sigma$-POROSO (CONJUNTO) - Definição 4.1.

t-POROSO (CONJUNTO) - Definição 4.3.

$\sigma$-t-POROSO (CONJUNTO) - Definição 4.3.

RAIO DE CONVERGÊNCIA - de uma função holomorfa $f: U$ (aberto) $\subset E \rightarrow \mathbb{C}$ ( $E$ Banach) num ponto $a \in U$ é dado por:

$r_{c} f(a)=\sup \{r>0$; a série de Taylor (de $f$ ) em torno de $a$ converge unif. em $B[a ; r]\}$.

RAIO DE LIMITAÇÃO - de uma função holomorfa $f: U$ (aberto) $\subset E \rightarrow \mathbb{C}(E$ Banach) num ponto $a \in U$ é dado por:

$$
r_{b} f(a)=\sup \{r>0 ; B[a ; r] \subset U \text { e } f \text { é limitada em } B[a ; r]\} .
$$

RARO (CONJUNTO) - $A \subset X$ (espaço topológico) é raro se, e somente se, seu fecho tem interior vazio $(\operatorname{int} \bar{A}=\phi)$.

SEGUNDO-CONTÁVEL (ESPAÇO) - um espaço topológico é segundo-contável quando sua topologia admite uma base enumerável de abertos.

SUPERCÍCLICO (VETOR) - Definição 1.11.

TIPO FINITO (POLINÔMIO DE) - sobre um espaço de Banach $E$ é um polinômio $p: E \rightarrow \mathbb{C}$, dado por $p=p_{0}+p_{1}+\ldots+p_{m}$, sendo cada $p_{k}(k=0,1, \ldots, m)$ do tipo:

$$
p_{k}(x)=\sum_{j=1}^{l} c_{j} \cdot \varphi_{j}(x)^{k} \quad\left(\varphi_{j} \in E^{\prime}, c_{j} \in \mathbb{C}\right) .
$$

TRANSLAÇÃO NÃO-NULA - translação por um vetor não-nulo.

UNIVERSALIDADE - Definição 1.1.

VIZINHANÇA UNIFORME - de um conjunto $A \subset E$ (Banach) é um conjunto do tipo $A+B(0 ; \delta)$, para algum $\delta>0$.

WEIGHTED BACKWARD SHIFT - sobre um espaço de sequências $E$ é um operador $T: E \rightarrow E$ dado por $T\left(x_{1}, x_{2}, x_{3}, \ldots\right)=\left(a x_{2}, a x_{3}, a x_{4}, \ldots\right)$ (a escalar). Podemos considerar ainda um caso mais geral onde o peso $a$ varia em cada coordenada. 


\section{Referências}

[1] Aвakumov, E. E Gordon, J., Common hypercyclic vectors for multiples of backward shift, J. Funct. Anal. 200, no. 2 (2003), 494-504.

[2] ANSARI, S.I., Hypercyclic and cyclic vectors, J. Funct. Anal. 128 (1995), 374-383.

[3] AnSARI, S.I., Existence of hypercyclic operators on topological vector spaces, J. Funct. Anal. 148 (1997), 384-390.

[4] Aron, R., Weakly uniformly continuous and weakly sequentially continuous entire functions, Advances in holomorphy (Proc. Sem. Univ. Fed. Rio de Janeiro, Rio de Janeiro, 1977), North-Holland Math. Studies, 34. North-Holland, Amsterdam (1979), 47-66.

[5] Aron, R. e Markose, D., On universal functions, J. Korean Math Soc. 41, no. 1 (2004), 65-76.

[6] Aron, R., Seone-Sepúlveda, J.B. E Weber, A., Chaos on function spaces, Bull. Austral. Math. Soc., 71 (2005), 411-415.

[7] BAyART, F., Common hypercyclic vectors for composition operators, J. Operator Theory 52, no. 2 (2004), 353-370.

[8] Bernal-González, L., On hypercyclic operators on Banach spaces, Proc. Amer. Math. Soc. 127 (1999), 1003-1010.

[9] Bernal-GonzÁLez, L., Universal images of universal elements, Studia Math. 138 (3) (2000), 241-250. 
[10] Bernal-González, L. E Montes-RodríGuez, A., Non-finite dimensional closed vector spaces of universal functions for composition operators, Journal of Approximation Theory 82 (1995), 375-391.

[11] BÈs, J., Invariant manifolds of hypercyclic vectors for the real scalar case, Proc. Amer. Math. Soc. 127 (1999), 1801-1804.

[12] Bès, J. E Peris, A., Hereditarily hypercyclic operators, J. Funct. Anal. 167 (1999), 94-112.

[13] Birkhoff, G., Démonstration d'un théoréme elémentaire sur les fonctions entiéres, Cr. R. Acad. Sci. Paris 189 (1929), 473-475.

[14] Bonet, J. E PeRIS, A., Hypercyclic operators on non-normable Fréchet spaces, J. Funct. Anal. 159 (1998), 587-595.

[15] Bourdon, P.S., Invariant manifolds of hypercyclic vectors, Proc. Amer. Math. Soc. 118 (1993), 845-847.

[16] Brunkalla, K.P., Perturbation of hypercyclic and supercyclic operators, Ph.D. Thesis, Kent State University (2001).

[17] Conway, J., A Course in Functional Analysis, Graduate Texts in Mathematics, 96. Springer-Verlag, New York (1990).

[18] Costakis, G. e Sambarino, M., Genericity of wild holomorphic functions and common hypercyclic vectors, Adv. Math. 182, no. 2 (2004), 278-306.

[19] Enflo, P., On the invariant subspace problem for Banach spaces, Acta Math. 158 (1987), 213-313.

[20] Fernández, G. e Hallack, A.A., Remarks on a result about hypercyclic nonconvolution operators, J. Math. Anal. Appl. 309, no. 1 (2005), 52-55.

[21] Gethner, R.M. e Shapiro, J.H., Universal vectors for operators on spaces of holomorphic functions, Proc. Amer. Math. Soc. 100 (1987), 281-288.

[22] Godefroy, G. e Shapiro, J.H., Operators with dense, invariant, cyclic vector manifolds, J. Funct. Anal. 98 (1991), 229-269. 
[23] Grivaux, S., Construction of operators with prescribed behaviour, Archiv der Math. 81 (2003), 291-299.

[24] Grivaux, S., Hypercyclic operators, mixing operators, and the Bounded Steps Problem, J. Operator Theory 54, no. 1 (2005), 147-168.

[25] Grosse-Erdmann, K.-G., Universal families and hypercyclic operators, Bull.(New Series) Amer. Math. Soc. 36, no. 3 (1999), 345-381.

[26] Herrero, D.A., Limits of hypercyclic and supercyclic operators, J. Funct. Anal. 99 (1991), 179-190.

[27] Kitai, C., Invariant closed sets for linear operators, Thesis, Univ. of Toronto, Toronto (1982).

[28] MacLane, G.R., Sequences of derivatives and normal families, J. Analyse Math. 2 (1952/53), 72-87.

[29] Megginson, R.E., An Introduction to Banach Space Theory, Graduate Texts in Mathematics, 183. Springer-Verlag, New York (1998).

[30] Montes-Rodríguez, A., Banach spaces of hypercyclic vectors, Michigan Math. J. 43 (1996), 419-436.

[31] MujicA, J., Complex Analysis in Banach Spaces, North-Holland Mathematics Studies, 120 - Notas de Matemática (107), 1986.

[32] Охтовy, J.C., Measure and Category (Second Edition), Graduate Texts in Mathematics, 2. Springer-Verlag, New York (1980).

[33] PÁL, J., Zwei kleine Bemerkungen, Tohoku Math. J. 6 (1914), 42-43.

[34] Rolewicz, S., On orbits of elements, Studia Math. 32 (1969), 17-22.

[35] Salas, H., Hypercyclic weighted shifts, Trans. Amer. Math. Soc. 347 (1995), 9931004.

[36] Seoane-Sepúlveda, J.B., Explicit constructions of dense common hypercyclic subspaces, Preprint. 